\title{
Neural Stem Cells of the Subventricular Zone Contribute to Neuroprotection of the Corpus Callosum after Cuprizone-Induced Demyelination
}

\author{
-Erica Butti, ${ }^{1}$ Marco Bacigaluppi, ${ }^{1,3}$ Linda Chaabane, ${ }^{1}$ Francesca Ruffini, ${ }^{1}$ Elena Brambilla, ${ }^{1}$ Giulia Berera,${ }^{3}$ \\ Carolina Montonati, ${ }^{3}$ Angelo Quattrini, ${ }^{2}$ and $\mathbb{C}^{-}$Gianvito Martino ${ }^{1,3}$ \\ ${ }^{1}$ Neuroimmunology Unit, Institute of Experimental Neurology, Division of Neuroscience, IRCCS San Raffaele Hospital, 20132 Milan, Italy, ${ }^{2}$ Neuropathology \\ Unit, Institute of Experimental Neurology, Division of Neuroscience, IRCCS San Raffaele Hospital, 20132 Milan, Italy, and ${ }^{3}$ Vita Salute San Raffaele \\ University, 20132 Milan, Italy
}

Myelin loss occurring in demyelinating diseases, including multiple sclerosis, is the leading cause of long-lasting neurological disability in adults. While endogenous remyelination, driven by resident oligodendrocyte precursor cells (OPCs), might partially compensate myelin loss in the early phases of demyelinating disorders, this spontaneous reparative potential fails at later stages. To investigate the cellular mechanisms sustaining endogenous remyelination in demyelinating disorders, we focused our attention on endogenous neural precursor cells (eNPCs) located within the subventricular zone (SVZ) since this latter area is considered one of the primary sources of new OPCs in the adult forebrain. First, we fate mapped SVZ-eNPCs in cuprizone-induced demyelination and found that SVZ endogenous neural stem/precursor cells are recruited during the remyelination phase to the corpus callosum (CC) and are capable of forming new oligodendrocytes. When we ablated SVZ-derived eNPCs during cuprizone-induced demyelination in female mice, the animals displayed reduced numbers of oligodendrocytes within the lesioned CC. Although this reduction in oligodendrocytes did not impact the ensuing remyelination, eNPC-ablated mice experienced increased axonal loss. Our results indicate that, in toxic models of demyelination, SVZderived eNPCs contribute to support axonal survival.

Key words: ablation; cuprizone; multiple sclerosis; neural stem cells; sub ventricular zone; transgenic mice

Significance Statement

One of the significant challenges in MS research is to understand the detrimental mechanisms leading to the failure of CNS tissue regeneration during disease progression. One possible explanation is the inability of recruited oligodendrocyte precursor cells (OPCs) to complete remyelination and to sustain axonal survival. The contribution of endogenous neural precursor cells (eNPCs) located in the subventricular zone (SVZ) to generate new OPCs in the lesion site has been debated. Using transgenic mice to fate map and to selectively kill SVZ-derived eNPCs in the cuprizone demyelination model, we observed migration of SVZ-eNPCs after injury and their contribution to oligodendrogenesis and axonal survival. We found that eNPCs are dispensable for remyelination but protect partially from increased axonal loss.

\section{Introduction}

Demyelination is the classical hallmark of multiple sclerosis (MS), featuring the degeneration of myelin sheets as well as of

Received Jan. 22, 2018; revised March 20, 2019; accepted March 21, 2019.

Author contributions: E. Butti, M.B., and G.M. designed research; E. Butti, L.C., F.R., E. Brambilla, G.B., C.M., and A.Q. performed research; E. Butti, M.B., L.C., and G.M. analyzed data; E. Butti, M.B., and G.M. wrote the paper.

This work was supported by FISM (Federazione Italiana Sclerosi Multipla) 2013/R/4, TargetBrain (EU Framework 7 project HEALTH-F2-2012-279017). M.B. has been supported by the Ministero della Salute Italiana (Progetto Giovani Ricercatori 58/GR-2011-02348160). We thank L. Muzio, L. Ottoboni, G. Gullotta, and S. Bezukladova for critical comments and corrections; and the Advanced Light and Electron Microscopy Biolmaging Center, and Centro di Imaging Sperimentale (Experimental Imaging Centre), San Raffaele Scientific Institute.

The authors declare no competing financial interests. oligodendrocytes cell bodies. Demyelinated plaques define a well demarcated hypocellular area characterized by loss of myelin, relative preservations of axons and formation of astrocytic scars (Noseworthy et al., 2000). A certain degree of spontaneous remyelination, which offers new hope for the development of innovative neuroprotective strategies, accompanies the early phases of the disease. However, this endogenous mechanism of repair shows limited efficiency and high heterogeneity in patients. Epi-

Correspondence should be addressed to Gianvito Martino at martino.gianvito@hsr.it or Erica Butti at butti.erica@hsr.it.

https://doi.org/10.1523/JNEUROSCI.0227-18.2019

Copyright $\odot 2019$ the authors 
sodes of spontaneous remyelination occur in MS lesions; indeed, $19 \%$ of lesions are partially remyelinated, while in $23 \%$ of cases the remyelination is complete (Barkhof et al., 2003). Loss of oligodendrocyte progenitor cells (OPCs) is undoubtedly among the leading causes for hampering endogenous remyelination, along with the limited ability of these cells to migrate toward demyelinating lesions and to differentiate into mature oligodendrocytes (Franklin and Ffrench-Constant, 2008). Insufficient clearing of myelin debris also affects the generation of new myelin sheaths (Skripuletz et al., 2013).

Parenchymal OPCs naturally sustain remyelination (Goldman, 2005; Tripathi et al., 2010; Zawadzka et al., 2010), but experimental evidence shows that endogenous neural precursor cells (eNPCs) of the subventricular zone (SVZ) are able to generate remyelinating cells in both physiological and injury conditions (Nait-Oumesmar et al., 1999; Picard-Riera et al., 2002; Aguirre et al., 2007; Jablonska et al., 2010; Gonzalez-Perez and Alvarez-Buylla, 2011). Endogenous NPCs sustain remyelination through the following: (1) proliferation and differentiation in OPCs followed by oligodendrocyte maturation; (2) release of neurotrophic cytokines/growth factors (e.g., leukemia inhibitory factor, IGF-1, FGF-2) that act on the survival of local oligodendrocytes; or (3) in an indirect way, by modulating astrocytes and microglia at the lesion site (Kipp et al., 2009). Indeed, fatemapping studies demonstrated that, beside parenchymal OPCs (pOPCs), eNPCs are also enrolled in the remyelinating process occurring after CNS damage. Accordingly, both pOPCs and eNPCs contribute to remyelination in the cuprizone-mediated demyelination model, highlighting regional differences along the anteroposterior axis and a context-dependent contribution of both populations (Steelman et al., 2012; Xing et al., 2014; Brousse et al., 2015).

The specific contribution of pOPCs or eNPCs during a demyelination event and whether one population of cells is more important than the other one still remain unknown.

In this study, we examined the specific role of SVZ-eNPCs in a model of demyelination induced by cuprizone, using the transgenic mouse line Nestin-CreER ${ }^{\mathrm{T} 2}$-YFP (Imayoshi et al., 2006), which is suitable for fate mapping and the NestinTK (Butti et al., 2012) in which SVZ-eNPCs can be selectively ablated upon ganciclovir (GCV) treatment. The cuprizone model has been demonstrated to have a high potential to study demyelination and remyelination, although its inflammatory cellular component differs from the one in MS (Acs et al., 2009). We here show that SVZ-eNPCs can migrate along the corpus callosum (CC) after cuprizoneinduced injury. eNPCs contribute to the number of mature oligodendrocytes but are dispensable for the remyelination process. However, eNPC loss is accompanied by a partial increase of axonal loss in the CC after cuprizone-induced damage.

\section{Materials and Methods}

Study approval and animals. Adult female C57BL/6 mice ( $6-8$ weeks old) and transgenic mice were purchased from Charles River (https://www. criver.com/products-services/find-model/c57bl6-mouse?region=27) or were generated in our animal facility in SPF conditions. Experimental procedures, performed blindly, were approved by the Institutional Animal Care and Use Committee (no. 570 and 750) of the San Raffaele Scientific Institute (Milan, Italy). To perform fate mapping and assess the behavior of the eNPCs during remyelination, we used Nestin-CreER ${ }^{\mathrm{T} 2}$ line 5-1 transgenic mice provided by Dr. Ryoichiro Kageyama (Tokyo University, Japan) (Imayoshi et al., 2006) expressing a CreER ${ }^{\mathrm{T} 2}$ fusion protein under the control of the rat Nestin gene promoter. These mice were crossed with a reporter mouse line, Rosa26-eYFP (Srinivas et al., 2001 ), to produce the Nestin-CreER ${ }^{\mathrm{T} 2}$ line 5-1: Rosa26-eYFP transgenic mice (from here on called Nestin:YFP). To ablate SVZ eNPCs selectively, we used the NestinTK transgenic mouse line, in which the thymidine kinase $(t k)$ gene, a suicide gene, is under the control of the second intron of Nestin rat promoter (Butti et al., 2012). Treatment of NestinTK mice with ganciclovir for 4 weeks, by subcutaneously implanted osmotic minipump, allowed ablation of Nestin ${ }^{+}$eNPCs. To verify the ablation of cells derived from SVZ, we used a triple transgenic mouse line, which we obtained by crossing Nestin:YFP with NestinTK mice (from here on called Nestin:YFP-NestinTK).

Experimental design. In the first experimental setting, to understand the mobilization and differentiation of SVZ-eNPCs after cuprizoneinduced demyelination, animals were housed under standard laboratory conditions with food and water available ad libitum. Recombination in Nestin-CreER ${ }^{\mathrm{T} 2}$ : Rosa26-eYFP transgenic mice ( $n=6$ /group) was induced by oral gavage of tamoxifen (TAM; catalog \#T5648, SigmaAldrich; https://www.sigmaaldrich.com/catalog/product/sigma/t5648? lang =it\&region=IT) delivered at a dose of $0.3 \mathrm{~g} / \mathrm{kg} / \mathrm{d}$ for 4 consecutive days. Tamoxifen was prepared at $40 \mathrm{mg} / \mathrm{ml}$ in corn oil (Sigma-Aldrich). Vehicle controls ( $n=6$ /group) received corn oil without tamoxifen (called Oil/control mice from here on). Tamoxifen-treated mice were fed with $0.2 \%$ cuprizone (w/w; biscyclohexanoneoxaldihydrazone; https:// www.sigmaaldrich.com/catalog/product/sigma/c9012?lang=it\&region=IT; \#c9012; Sigma-Aldrich) mixed in standard rodent chow for 6 consecutive weeks, starting 1 week after tamoxifen administration (called TAM/ cuprizone from here on). Mice returned to a normal diet for a period of 2 more weeks, and they were killed at 4 and 8 weeks from the diet start. As a control, we used Nestin:YFP mice treated with tamoxifen but fed normal food (called TAM/control from here on).

In the second experimental setting, to study the role of the SVZ-eNPC population during remyelination, NestinTK ${ }^{+}$mice, with ablated cells, and NestinTK ${ }^{-}$mice ( $n=6 /$ group), in the presence of cells, were fed $0.2 \%$ cuprizone mixed in standard rodent chow for 6 consecutive weeks. Then they returned to a normal diet for a period of 2 more weeks. Mice were killed afterward.

For the ganciclovir (http://www.ilprontuariofarmaceutico.it/dettagli. php?aic $=032837015 \mathrm{GCV}$; Citovirax, Roche) administration, we used two different protocols.

In the first protocol, we aimed at investigating the effect of SVZ-eNPC ablation during cuprizone-induced demyelination; thus, mice received $100 \mathrm{mg} / \mathrm{kg} / \mathrm{d}$ GCV dissolved in double-distilled $\mathrm{H}_{2} \mathrm{O}\left(\mathrm{ddH}_{2} \mathrm{O}\right)$ administered by subcutaneously implanted osmotic minipumps (ALZET model 2002, DURECT Corporation; http://www.alzet.com/ downloads/2002specs.pdf), as previously described (Garcia et al., 2004). GCV was administered for 4 weeks, starting from the first week after the beginning of the cuprizone diet. Implanted osmotic minipumps were replaced with a new minipumps after $14 \mathrm{~d}$ for a total of $28 \mathrm{~d}$ of treatment.

In the second protocol, we aimed to examine the effect of SVZ-eNPC ablation during remyelination after cuprizone-induced demyelination. GCV was administered for 4 weeks, starting from the fourth week after the beginning of the cuprizone diet. Implanted osmotic minipumps were replaced as in the first protocol.

NestinTK mice fed cuprizone and treated with GCV are named NestinTK ${ }^{+}$GCV-Cup, while NestinTK mice fed cuprizone and treated with PBS are called NestinTK ${ }^{+}$PBS-Cup. As a control, we used C57BL/6 mice fed cuprizone and treated with GCV (called NestinTK ${ }^{-}$GCV-Cup mice).

In the second experimental setting, to verify the ablation of cells derived from SVZ after the GCV administration, we treated Nestin:YFPNestinTK with tamoxifen by oral gavage for 4 consecutive days. One week after tamoxifen administration, mice were fed $0.2 \%$ cuprizone (w/w; biscyclohexanoneoxaldihydrazone) mixed in standard rodent chow for 6 consecutive weeks. Then they returned to a normal diet for 2 weeks. GCV was administered over a period of 4 weeks, starting from the fourth week after the beginning of the cuprizone diet. Afterward, mice were killed. As a control, we used Nestin:YFP-NestinTK mice treated with tamoxifen and PBS and fed cuprizone.

Tissue pathology. Mice were deeply anesthetized by intraperitoneal injection of Avertin (2.5 g of 2,2,2 tribromoethanol; catalog \#T48402 Sigma-Aldrich), dissolved in $5 \mathrm{ml}$ of 2-metil-2-butanol (Sigma-Aldrich) 
and $200 \mathrm{ml}$ of $\mathrm{ddH}_{2} \mathrm{O}$. The toe pinch response method was used to determine the depth of anesthesia. Mice were first transcardially perfused with a saline buffer $(0.9 \% \mathrm{NaCl})$ plus EDTA [500 $\mu$ l of EDTA $0.5 \mathrm{M}$ (Sigma-Aldrich) were added to $250 \mathrm{ml}$ of saline buffer] at room temperature (RT) until there was no blood visible in the liver and then with $4 \%$ paraformaldehyde (PFA; catalog \#158127, Sigma-Aldrich) in PBS $1 \times$ to preserve tissue in a life-like state. Brains were removed and postfixed in $4 \%$ PFA overnight at $+4^{\circ} \mathrm{C}$. On the following day, brains were washed in PBS $3 \times$ for $5 \mathrm{~min}$ and cryopreserved in $30 \%$ sucrose (Sigma-Aldrich) in PBS $1 \times$. After $48 \mathrm{~h}$, brains were embedded in Tissue-Tek OCT Compound (BioOptica) and frozen in isopentane in a liquid nitrogen bath. Brains were stored at $-80^{\circ} \mathrm{C}$ until sectioned. For pathology, immunohistochemistry, and immunofluorescence, coronal sections with a thickness of $14 \mu \mathrm{m}$ were obtained using a Leica cryostat (catalog \#CM1850), collected onto Superfrost slide, and air dried overnight before being stored at $-80^{\circ} \mathrm{C}$ until staining.

To evaluate myelin, slides were stained with Luxol fast blue (LFB) staining (catalog \#S3382, Sigma-Aldrich), a well established method to stain myelin and its phospholipids. Briefly, slides were dehydrated with increasing concentrations of ethanol $(50 \%, 70 \%$, and 100\%) and then left in $0.1 \%$ LFB solution (containing LFB, ethyl alcohol 95\%, and glacial acetic acid) overnight in the oven at $56^{\circ} \mathrm{C}$. The following day, slides were first rinsed with $96 \%$ ethanol and then with $\mathrm{ddH}_{2} \mathrm{O}$. White matter was differentiated from gray matter in $0.1 \%$ lithium carbonate solution; followed by $70 \%$ ethanol for $30 \mathrm{~s}$, then rinsed in $\mathrm{ddH}_{2} \mathrm{O}$. After that, slides were treated with $0.8 \%$ of periodic acid for $10 \mathrm{~min}$ and with Schiffs reagent (catalog \#3952016, Sigma-Aldrich) for $20 \mathrm{~min}$. Slides were rinsed with washing solution three times and then with $\mathrm{ddH}_{2} \mathrm{O}$ and were dehydrated with increasing concentrations of ethanol (50\%, 70\%, 100\%, and $100 \%)$. Slides were put in xylene for $4 \mathrm{~min}$ and then mounted with nonaqueous DPX solution (Sigma-Aldrich).

To detect axonal damage, slides were stained with Bielschowsky stain. Slides were washed $3 \times$ for $5 \mathrm{~min}$ in $\mathrm{ddH}_{2} \mathrm{O}$, then placed in $10 \%$ silver nitrate (catalog \#209139, Sigma-Aldrich) in the dark at RT for $20 \mathrm{~min}$ and then washed $2 \times$ for 5 min in $\mathrm{ddH}_{2} \mathrm{O}$. Concentrated ammonium hydroxide was added drop by drop to the silver nitrate solution kept from the previous step, and slides were incubated in this solution for $15 \mathrm{~min}$ in the dark at RT. Next, slides were washed in $\mathrm{ddH}_{2} \mathrm{O}$ with three to five drops of ammonium hydroxide for $2 \mathrm{~min}$ at RT. Developer solution $(0.2$ $\mathrm{ml}$ of $37 \%$ formaldehyde, $12 \mathrm{ml}$ of $\mathrm{dd}_{2} \mathrm{O}, 12.5 \mu \mathrm{l}$ of $20 \%$ nitric acid, and $0.05 \mathrm{~g}$ of citric acid) was added to the silver hydroxide solution saved from the previous step. Slides were stained in this solution for $10 \mathrm{~min}$ until they turned black, then were washed in $\mathrm{dd}_{2} \mathrm{O}$ for $2 \mathrm{~min}$, fixed in $5 \%$ sodium thiosulfate for 3-5 min, washed in $\mathrm{ddH}_{2} \mathrm{O}$, and dehydrated with solutions with increasing concentrations of ethanol $(50 \%, 70 \%$, $100 \%$, and $100 \%$ ). Slides were put in xylene for $4 \mathrm{~min}$ and then mounted with nonaqueous DPX solution.

For immunofluorescence, brain sections were air dried for at least 40 min and then rinsed $3 \times$ for 5 min with PBS $1 \times$. Nonspecific binding sites were blocked by incubation in the blocking solution (PBS $1 \times$ containing $0.1 \%$ Triton X-100, $10 \% \mathrm{FBS}, 1 \mathrm{mg} / \mathrm{ml} \mathrm{BSA}$ ) for $1 \mathrm{~h}$ at RT. Sections were then incubated overnight at $+4^{\circ} \mathrm{C}$ with the appropriate primary antibody diluted in blocking solution in a humidity chamber. The following primary antibodies were used: goat anti-DCX (doublecortin; 1:100; catalog \#sc-8066, Santa Cruz Biotechnology; RRID:AB_2088494); rat antiBrdU (1:100; catalog \#sc-8066, Santa Cruz Biotechnology; RRID: AB_2088494); mouse anti-Olig2 (oligodendrocyte transcription factor 2; 1:200; catalog \#MABN50, Millipore; RRID:AB_10807410); mouse antiGFAP (glial fibrillary acidic protein; 1:300; catalog \#MAB12029, Immunological Sciences); rabbit anti-Iba-1 (ionized calcium binding adaptor molecule 1; 1:400; catalog \#019-19741, Wako; RRID:AB_839504); chicken anti-YFP (1:300; catalog \#ab13970, Abcam; RRID:AB_300798); rabbit antiALDH1 (1:200, Abcam catalog \#ab87117, RRID:AB_10712968), hamster anti-CD11c (1:75; catalog \#BNB110-97871, Novus Biologicals); mouse antiCD68 (1:200; catalog \#ab31630, Abcam; RRID:AB_1141557); mouse anti-LPL (1:100; catalog \#ab21356, Abcam; RRID:AB_446221); rat antiLy6C (1:100; catalog \#ab15627, Abcam; RRID:AB_302004); and mouse anti-APP (1:100; catalog \#A8717, Sigma-Aldrich; RRID:AB_258409). For double labeling, some primary antibodies were incubated simulta- neously. The next day, slides were washed $3 \times$ for $5 \mathrm{~min}$ in PBS $1 \times$ and then incubated for $1 \mathrm{~h}$ at RT in the dark with appropriate fluorophoreconjugated secondary antibodies [Alexa Fluor 488, Alexa Fluor 546, and Alexa Fluor 633; 1:1000; catalog \#A-11006, Thermo Fisher Scientific (RRID:AB_2534074); catalog \#A-11055 (RRID:AB_2534102); catalog \#A-11001 (RRID:AB_2534069); catalog \#A-11008 (RRID:AB_143165); and catalog \#A-11003 (RRID:AB_2534071)]. In all immunofluorescence staining, nuclei were stained with DAPI (1:25,000; Roche) in PBS $1 \times$ for $1 \mathrm{~min}$. Sections were mounted with Dako fluorescent mounting medium and subjected to fluorescence and confocal microscope analysis.

For immunohistochemistry, brain sections were air dried for at least $40 \mathrm{~min}$ and then rinsed $3 \times$ for $5 \mathrm{~min}$ with PBS $1 \times$. The slides were boiled in a citrate buffer for $5 \mathrm{~min}$ ( $10 \mathrm{~mm}$ citric acid monohydrate, $\mathrm{pH}$ 6) and then cooled down to RT. Slides were washed in PBS $1 \times$ for $5 \mathrm{~min} 3 \times$ and then incubated in $0.3 \% \mathrm{H}_{2} \mathrm{O}_{2}$ in PBS $1 \times$ for $10 \mathrm{~min}$. To prevent unspecific binding, sections were incubated with blocking solution for $1 \mathrm{~h}$ at $\mathrm{RT}$. The sections were then incubated overnight at $+4^{\circ} \mathrm{C}$ with the primary antibody mouse anti-Olig2 (1:200; catalog \#MABN50, Millipore; RRID:AB_10807410) and mouse anti-APC (adenomatous polyposis coli; 1:100; catalog \#OP80, Calbiochem; RRID:AB_2057371), and were diluted in blocking solution in a humidity chamber. The next day, the slides were rinsed $3 \times$ for 5 min in PBS $1 \times$ and then incubated for $1 \mathrm{~h}$ at RT with anti-mouse biotinylated secondary antibody (1:500; catalog \#BA-9200, Vector Laboratories; RRID:AB_2336171) diluted in blocking solution. Sections were then rinsed and further incubated with an avidin-biotin complex (ABC reagent; catalog \#PK-6100, Vector Laboratories; RRID: AB_2336819) for $1 \mathrm{~h}$ at RT. The solution was prepared $1 \mathrm{~h}$ before use, as follows: $10 \mu \mathrm{l}$ of solution A $+10 \mu \mathrm{l}$ of solution B in $1 \mathrm{ml}$ of PBS $1 \times$. Slides were again washed for 5 min $3 \times$ in PBS $1 \times$, incubated in diaminobenzidine (DAB) solution (DAB Kit; catalog \#SK-4100, Vector Laboratories; RRID:AB_2336382) for $1 \mathrm{~min}$, washed in $\mathrm{dd}_{2} \mathrm{O}$, and then dehydrated with increasing concentrations of ethanol (50\%, 70\%, 100\%, 100\%). Finally, slices were put in xylene for $4 \mathrm{~min}$ and then mounted with nonaqueous DPX. Staining omitting the primary antibody was always used as negative control.

Determination of demyelination. To determine the demyelination in the corpus callosum, sections stained with Luxol fast blue were scored in a blinded manner and graded on a scale from 0 (normal myelin) to 3 (complete demyelination).

Neurolucida, confocal microscopy, and image analysis. Immunohistochemical staining was acquired on a Leica microscope using a $2.5 \times$ objective and were analyzed with Neurolucida software version 5.0. Three or four coronal sections were examined for each animal, including the rostral and caudal regions of the CC. Digital images $(N=3)$ of each coronal section were taken; in particular, images were captured within the medial and the lateral regions of the CC.

Images were obtained with a confocal microscope (SP5 and SP8, Leica; equipped with $40 \times$ objective) to analyze immunofluorescence staining. Two coronal sections were examined for each animal in rostral as well as in caudal regions of the CC. Digital images $(N=3-9)$ of each coronal section were taken; the images were captured within the medial and lateral regions of the CC. Analyses of images were performed using Photoshop CS4 software (Adobe Systems) or ImageJ software (NIH) or InForm Cell Analyzer (PerkinElmer) for cellular density and quantitative analysis. Quantitative cell count analysis was performed in a blinded manner.

In vivo magnetic resonance image. Noninvasive in vivo magnetic resonance imaging (MRI) techniques with high spatial resolution are a sensitive tool to monitor CC damage in mice exposed to cuprizone. For this purpose, we have optimized the protocol on our 7 tesla BioSpec preclinical scanner (Bruker BioSpin) to evaluate the extension of CC damage with T2-weighted MRI contrast and to access the degree of demyelination and axonal loss using diffusion tensor imaging (DTI). When performing in vivo MRI, animals were maintained under anesthesia, with a mixture of fluorane and $\mathrm{O}_{2}$ gas, and were placed in a bed with an integrated heating system to keep the body temperature at $37^{\circ} \mathrm{C}$. A surface coil dedicated to mouse brain imaging was used to allow high signal quality and to acquire highly resolved images with the following sequences: turbo spin-echo for T2-weighted images $\left(\mathrm{TR}=3 \mathrm{~s}, \mathrm{TE}=36 \mathrm{~ms}, 86 \times 85 \mu \mathrm{m}^{2}\right.$, 
A
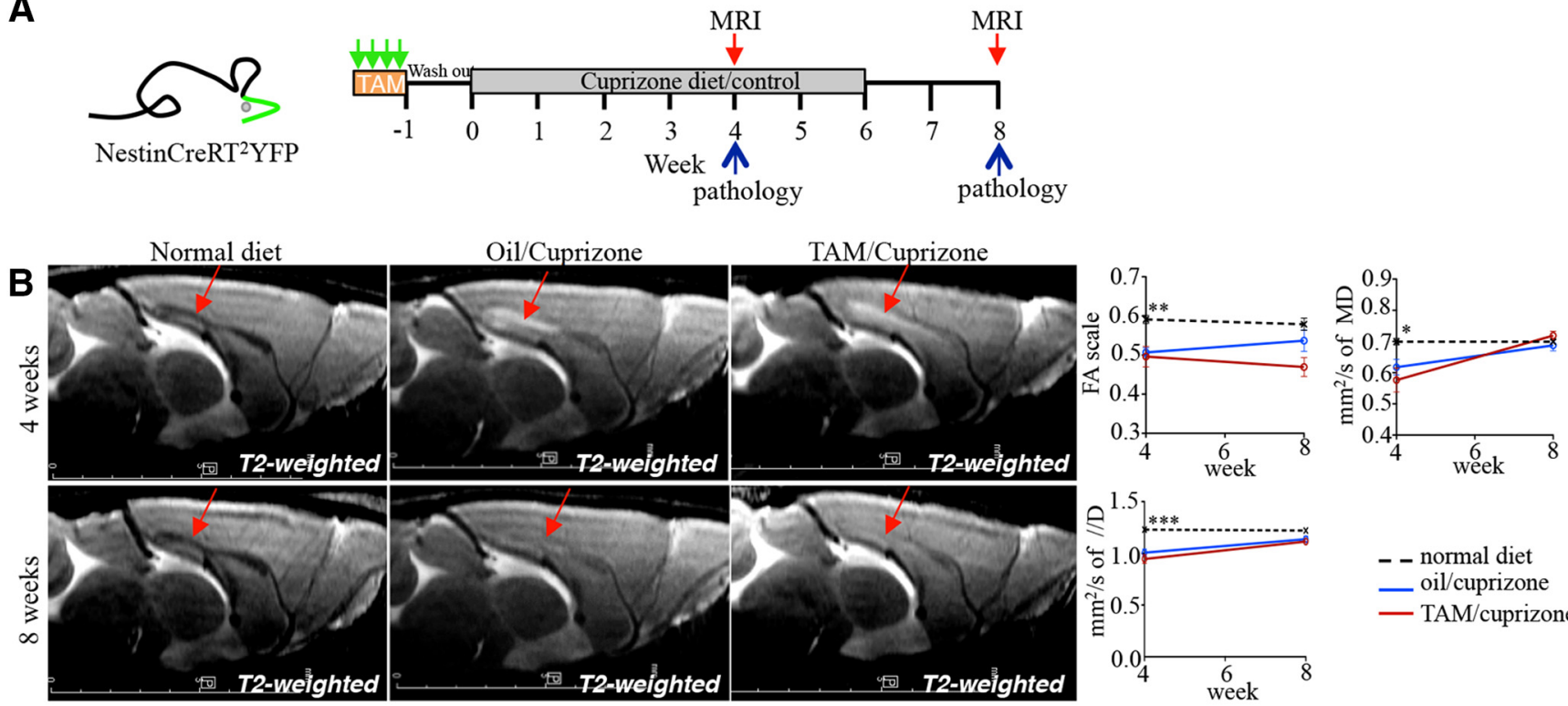

\section{- - normal diet \\ _ oil/cuprizone \\ — TAM/cuprizone}
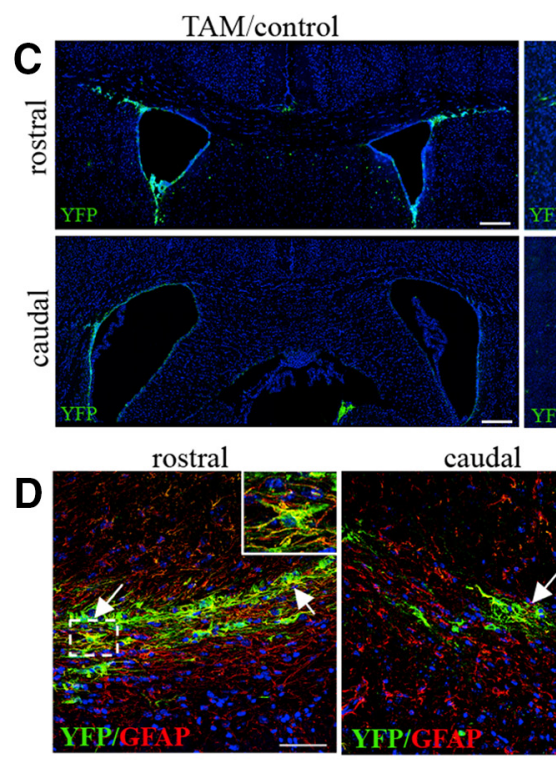

$F$
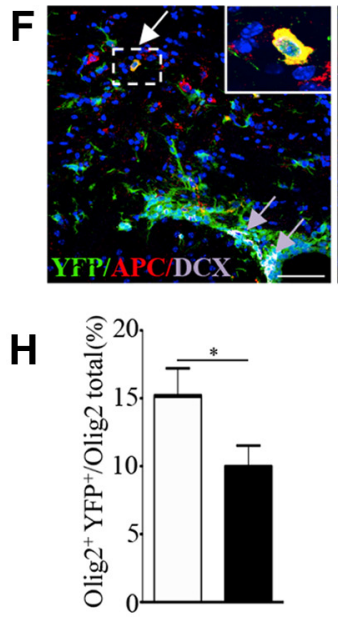

TAM/cuprizone 4 weeks

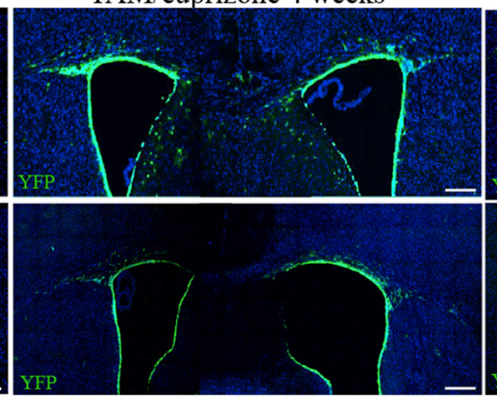

rostral
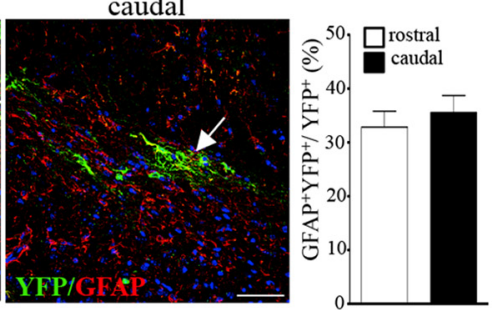

E
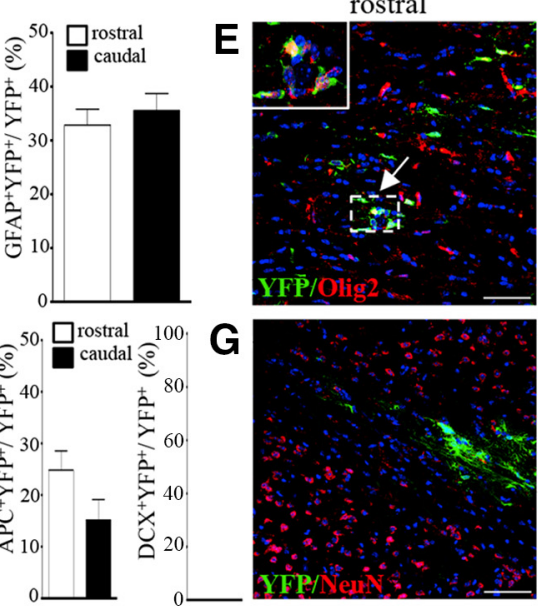

G
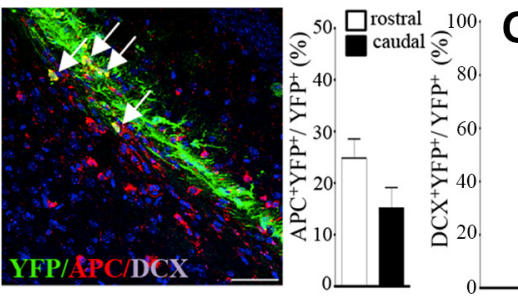

TAM/cuprizone 8 weeks
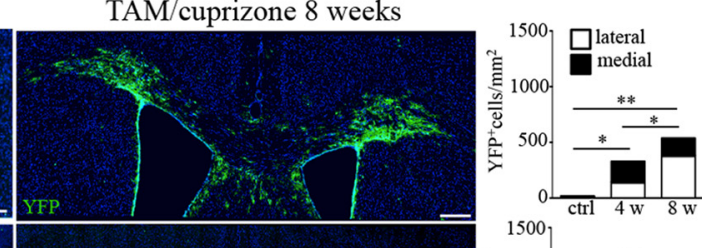

1500

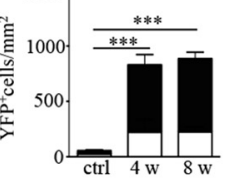

caudal
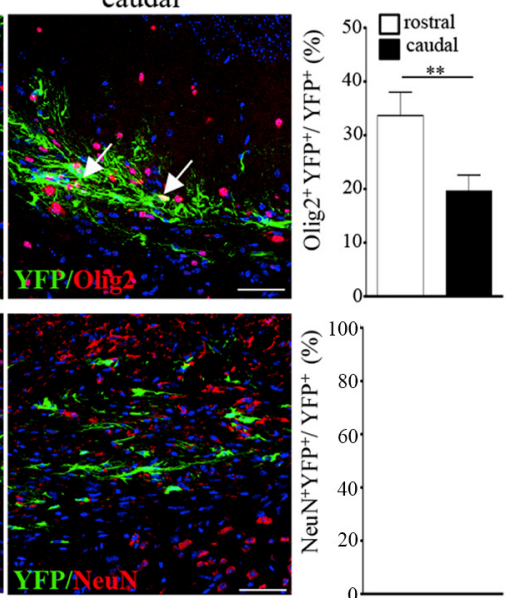

Figure 1. Cuprizone-induced demyelination in the corpus callosum. $A$, Experimental study design indicates the administration schedule of tamoxifen/oil via gavage and cuprizone diet, MRI analysis and tissue collection in Nestin:YFP mice. $\boldsymbol{B}$, Analysis of CC damage by in vivo MRI. Representative sagittal T2-weighted images in mice treated either with a normal or cuprizone diet and treated with either oil or TAM via gavage. The graphs represent the quantification of the lesion of the $C($ by DTI (FA, MD, and //D). Arrows indicate the hyperintense demyelinating lesions occurring in the corpus callosum. $n=3$ animals/group. C, Representative images and quantification of the mobilization of YFP ${ }^{+}$cells in Nestin:YFP mice treated with cuprizone for 4 or 8 weeks at the level of the rostral and caudal CC considering the lateral and medial area. Shown are representative images: YFP ${ }^{+}$cells are in green and nuclei stained by DAPI are in blue. (Figure legend continues.) 
sagittal section with a thickness of $0.8 \mathrm{~mm}$ ) and an echoplanar imaging method for DTI with 30 diffusion-weighted directions ( $\mathrm{b}$-values $=0$ and $950 \mathrm{~s} / \mathrm{mm}^{2}, \mathrm{TR} / \mathrm{TE}=3.4 \mathrm{~s} / 22 \mathrm{~ms}, 133 \times 113 \mu \mathrm{m}^{2}$, thickness $=0.8 \mathrm{~mm}$; Zhang et al., 2012; Tagge et al., 2016). All MRI data have been analyzed using the Paravision software package (BioSpec, Bruker BioSpin) to extract within the manually delimitated CC region $\left(0.31 \mathrm{~mm}^{2} \times\right.$ thickness of $0.8 \mathrm{~mm}$ ) the diffusivity parameters as the fractional anisotropy (FA), an index of fibers integrity; the axial diffusivity (//D), sensitive to axonal injury; and the mean diffusivity (MD), an index of myelinated axons.

Electron microscopy. For electron microscopy (EM) studies, ketamine/ xylazine-anesthetized mice were perfused transcardially with $0.9 \%$ saline, followed by Karnovsky's fixative (2\% PFA and 2.5\% glutaraldehyde; Sigma-Aldrich). Tissues were postfixed in osmium tetroxide for $2 \mathrm{~h}$, dehydrated in ascending alcohol concentrations, and embedded in EPON (Fluka). Ultrathin sections were mounted onto slot grids for viewing using a LEO 912 Transmission Electron Microscope, as previously described (Pluchino et al., 2003).

Statistical analysis. For statistical analyses, we used a standard software package (Prism version 5.00, GraphPad Software). Data were evaluated by unpaired $t$ test (for comparisons between two groups) or by one-way ANOVA followed by post hoc analysis (for comparisons among three groups), as indicated in the figure legends. The significance level was established at $p=0.05$. Whenever a treatment-time interaction or treatment effect was present, a post hoc analysis was performed by Bonferroni correction. For the score of Luxol fast blue analysis, we used a nonparametric statistical test, the Kruskal-Wallis test (for comparisons among three groups).

\section{Results}

\section{Mobilization and differentiation of SVZ-eNPCs in CC after cuprizone-induced damage}

To investigate the role of SVZ-derived eNPCs in generating new oligodendrocytes and to evaluate their contribution to remyelination after cuprizone-induced damage, we performed fate mapping using the Nestin-CreER ${ }^{\mathrm{T} 2}$-Rosa26-eYFP mouse line (Srinivas et al., 2001; Imayoshi et al., 2006). Recombination was induced treating Nestin:YFP mice with tamoxifen for 4 consecutive days, while controls received vehicle (corn oil). Seven days after the last tamoxifen injection, mice received either the cuprizone or the standard diet for 6 consecutive weeks. At the end of the cuprizone treatment, they returned to the standard diet and were then studied by MRI at the fourth and eighth week, and by histology at the eighth week (Fig. 1A, experimental paradigm).

MRI was performed at the fourth week when demyelination should be at its peak, and at the eighth week during the remyelination phase (Gudi et al., 2014). At the fourth week, the T2weighted signal of CC was hyperintense in cuprizone-treated mice, either treated or not treated with tamoxifen, suggesting myelin loss, while mice receiving the standard diet were unaffected. At the same time point, diffusivity parameters, such as FA, $\mathrm{MD}$, and //D were significantly decreased in cuprizone-treated

\section{$\leftarrow$}

(Figure legend continued.) $\quad n=6$ mice/group. $\boldsymbol{D}-\mathbf{G}$, Cell fate mapping of the differentiation fate of YFP ${ }^{+}$cells during the remyelination at 8 weeks after cuprizone challenge, in the Nestin: YFP mouse: representative coronal sections and quantification, in the rostral and caudal regions of $C C$, of the differentiation of YFP ${ }^{+}$cells (in green) either in astrocyte GFAP (red; $D$ ), in oligodendrocyte precursors 0lig2 (red; $\boldsymbol{E})$, in mature oligodendrocytes (APC; red; $\boldsymbol{F}$ ), or in neurons (NeuN in red; $G$ ). Nuclei stained by DAPI are in blue. Data are expressed as the percentage of double-positive cells on the total of YFP ${ }^{+}$cells. $n=6$ pictures $/ 6$ mice/area. Cells indicated by arrows in $\boldsymbol{D}-\boldsymbol{F}$ are double-positive cells and are magnified in the contoured inset; in $\boldsymbol{F}$, the purple arrows indicate the $\mathrm{DCX}{ }^{+}$cells. $\boldsymbol{H}$, Percentage of 0 lig2 ${ }^{+}$YFP ${ }^{+}$cells on the total of Olig2 ${ }^{+}$and the percentage of $\mathrm{APC}^{+}{ }^{+} \mathrm{FPP}^{+}$cells on the total of $\mathrm{APC}^{+}$cells in the rostral (white bar) and caudal (black bar) regions of CC. $n=6$ pictures $/ 6$ mice/area. Each bar represents the mean \pm SEM. ${ }^{*} p \leq 0.05 ;{ }^{* *} p \leq 0.01 ;{ }^{* * *} p \leq 0.001$ (unpaired $t$ test for $\boldsymbol{D}-\boldsymbol{F}$ and $\boldsymbol{H}$; one-way ANOVA followed by Bonferroni post hoc test for $\boldsymbol{B}$ and $\boldsymbol{C}$ ). Scale bars: $\boldsymbol{C}, 200 \mu \mathrm{m} ; \boldsymbol{D}-\mathbf{G}, 50 \mu \mathrm{m}$. mice. At the eighth week, during remyelination, these same parameters returned almost to normal values, as observed in mice receiving the standard diet (Fig. $1 B$ ).

Next, we fate mapped eNPCs using the Nestin:YFP mice, and we observed that $7 \mathrm{~d}$ after the last tamoxifen injection a large number of Nestin ${ }^{+}$NPCs were $\mathrm{YFP}^{+}$in the SVZ, whereas oiltreated mice rarely displayed $\mathrm{YFP}^{+}$cells, as previously described (Imayoshi et al., 2006). We then investigated the distribution and the number of $\mathrm{YFP}^{+}$cells in mice at the fourth and eighth weeks, focusing on two areas of the CC, the rostral and the caudal regions, mainly affected by the cuprizone-induced demyelination and considering the lateral and the medial areas. Oil/control and Oil/cuprizone mice exhibited only a very small fraction of YFP ${ }^{+}$ cells due to tamoxifen-independent recombination. TAM/cuprizone mice showed a consistent increase in the density of YFP ${ }^{+}$ cells compared with TAM/control mice already at the fourth week, which further significantly increased at the eighth week, mainly in the rostral region (Fig. 1C). In detail, we found that in the rostral region at 8 weeks, $\mathrm{YFP}^{+}$cells are more abundant in the lateral area compared with the medial area. On the contrary, in the caudal region, $\mathrm{YFP}^{+}$cells are more abundant in the medial compartment compared with the lateral area (Fig. 1C).

To study the fate of eNPCs we characterized the lineage identity of $\mathrm{YFP}^{+}$cells at the eighth week. We labeled sections for YFP from the TAM/cuprizone group with several cell type markers, namely the following: astrocytic marker GFAP, Olig2, the mature oligodendrocyte marker anti-APC, the neural precursor cell marker DCX, and the mature neuronal-specific nuclear protein $(\mathrm{NeuN})$. We observed that differentiation in astrocyte-like cells $\left(\mathrm{GFAP}^{+} \mathrm{YFP}^{+}\right)$was $30-35 \%$ of the total number of $\mathrm{YFP}^{+}$cells in both the rostral and the caudal CC (Fig. 1D). Differentiation in oligodendroglial lineage cells, Olig2 ${ }^{+} \mathrm{YFP}^{+}$cells, was instead more abundant in the rostral CC than in the caudal CC (Fig. 1E), accounting for $33.6 \pm 4 \%$ and $19.6 \pm 3 \%$ of total $\mathrm{YFP}^{+}$cells, respectively. Mature oligodendrocytes, namely $\mathrm{APC}^{+} \mathrm{YFP}^{+}$cells, were $15-20 \%$ of the total number of $\mathrm{YFP}^{+}$cells in both the rostral and the caudal CC (Fig. $1 F$ ). Surprisingly, in the CC we did not detect any $\mathrm{DCX}^{+} \mathrm{YFP}^{+}$cell (Fig. $1 F$ ) that was detectable in the SVZ. Even differentiation to neurons $\mathrm{NeuN}^{+} \mathrm{YFP}^{+}$was not detected (Fig. 1G). To clarify the contribution of the SVZ to oligodendrogenesis occurring after injury, we calculated the percentage of $\mathrm{YFP}^{+}$oligodendrocytes over the total number of oligodendrocytes. We found that at 8 weeks SVZ-derived Olig2 cells $\left(\mathrm{YFP}^{+}\right.$Olig2 $\left.{ }^{+}\right)$contribute to $15 \%$ and $10 \%$ of all Olig2 ${ }^{+}$ cells, in the rostral and caudal region of CC respectively, while SVZ-derived APC cells $\left(\mathrm{APC}^{+} \mathrm{YFP}^{+}\right)$contribute to $9 \%$ of all $\mathrm{APC}^{+}$cells in both regions (Fig. $1 H$ ).

\section{Early ablation of SVZ-eNPCs does not influence the progression of the damage and the remyelination in the corpus callosum}

We next investigated the role of the SVZ-derived eNPCs in the early phase of remyelination (Barkhof et al., 2003). We used mice carrying the TK gene under the control of the Nestin regulatory region to selectively ablate SVZ-confined eNPCs (Butti et al., 2012). NestinTK and their wild-type littermates (NestinTK ${ }^{-}$) were fed with cuprizone, as described above. One week after starting the cuprizone diet, mice were treated either with GCV or with vehicle (PBS) for 4 weeks to ablate eNPCs in the SVZ (Fig. 2A). Upon GCV treatment of NestinTK mice, we obtained a significant and robust ablation of eNPCs in the SVZ: neuroblasts labeled for DCX as well as transient amplifying cells, labeled by 
A
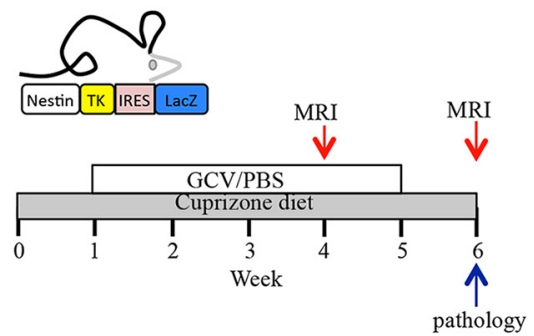
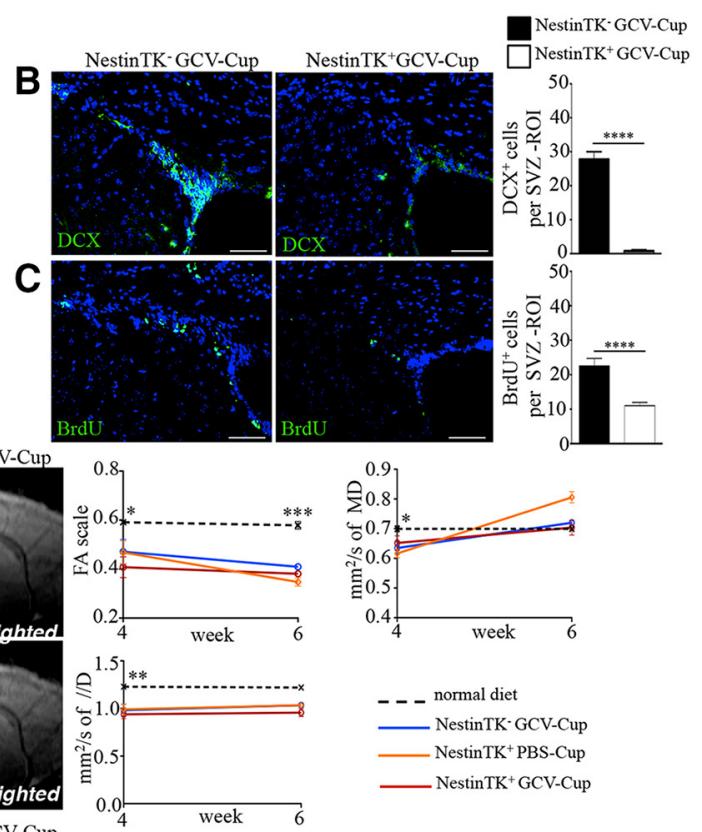

- - - normal diet

- NestinTK- GCV-Cup

NestinTK ${ }^{+}$PBS-Cup

NestinTK ${ }^{+}$GCV-Cup
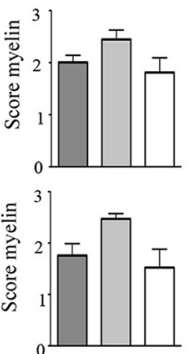

rostral region

$\square$ NestinTK·GCV-Cup

NestinTK ${ }^{+}$PBS-Cup

$\square$ NestinTK ${ }^{+}$GCV-Cup

F
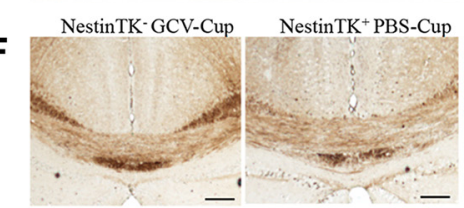

NestinTK+ GCV-Cup
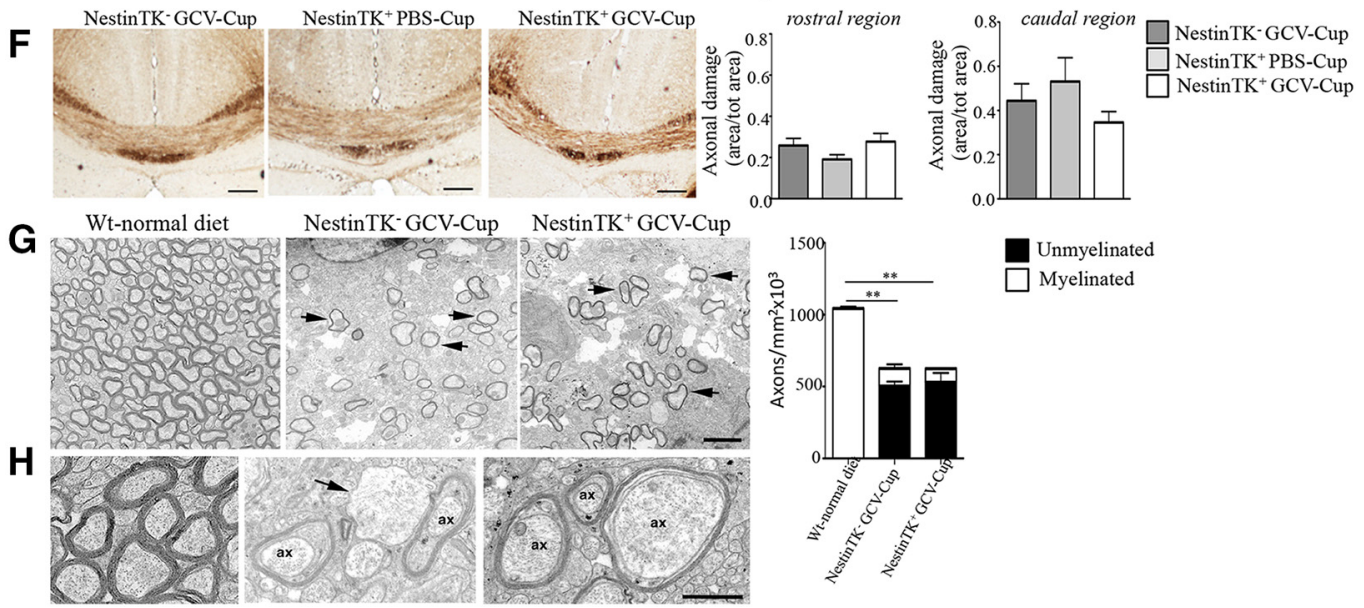

Unmyelinated

$\square$ Myelinated

Figure 2. Demyelination after cuprizone treatment is unaltered after ablation of eNPCs. $A$, Experimental design: NestinTK ${ }^{+}$mice or controls, NestinTK ${ }^{-}$, were treated with either GCV or PBS during cuprizone-induced demyelination of the CC. MRI studies were performed at 4 and 6 weeks, and pathology was performed at 6 weeks of diet. $B, C$, Representative images and quantification of the ablation of eNPC after 4 weeks of $\mathrm{GCV}$ treatment in NestinTK ${ }^{+}$mice at the sixth week of cuprizone treatment: neuroblasts were labeled for DCX in $\boldsymbol{B}$, transient amplifying cells for BrdU in $\boldsymbol{C}$. $n=3$ mice/group. D, Analysis of CC damage by in vivo MRI. Representative brain sagittal T2-weighted images acquired on NestinTK ${ }^{-}$GCV-Cup, NestinTK ${ }^{+}$PBS-Cup, and NestinTK ${ }^{+}$GCV-Cup mice at the fourth and sixth week of the cuprizone diet. Arrows indicate the demyelinating lesion of the corpus callosum that is quantified by the analysis of DTI parameters FA, MD, and //D in the graphs. $n=3$ mice/group. $\boldsymbol{E}$, Representative LFB-stained coronal brain sections at the level of the rostral and caudal $C$ C of NestinTK ${ }^{-}$and NestinTK ${ }^{+}$mice treated either with PBS or GCV at the sixth week of the cuprizone diet; the demyelination damage is quantified in the graphs on the right. Arrows indicate the demyelinating lesion of the corpus callosum. $n=8$ mice/group. $0=$ complete myelination, 3 = complete demyelination. $\boldsymbol{F}$, Representative brain sections of Bielschowsky staining are shown in the caudal region of $\mathrm{CC}$ in NestinTK ${ }^{-}$and NestinTK ${ }^{+}$mice treated with either PBS or GCV 2 weeks after the end of the cuprizone diet. The entity of axonal damage was evaluated in the rostral and caudal regions by Bielschowsky staining. $n=3$ mice/group. G, Representative electron micrographs of the caudal $\mathrm{CC}$ of mice treated with the normal diet, of NestinTK ${ }^{-} \mathrm{GCV}$-Cup and NestinTK ${ }^{+} \mathrm{GCV}$-Cup mice at the sixth week of the cuprizone diet. In the graph quantification of unmyelinated (in black) and myelinated (in white) axons in mice treated with the normal diet, NestinTK ${ }^{-} \mathrm{GCV}$-Cup and NestinTK ${ }^{+} \mathrm{GCV}$-Cup mice are shown. Arrows indicate the remyelinated axons. $n=3$ mice/group. $\boldsymbol{H}$, High-magnification image of representative myelinated, unmyelinated, and remyelinated axons. Arrow indicates the demyelinated axon. ax, Axon. Each bar represents the mean \pm SEM: ${ }^{*} p \leq 0.05 ;{ }^{* *} p \leq 0.01 ;{ }^{* * *} p \leq 0.001 ;{ }^{* * *} p \leq 0.0001$. One-way ANOVA followed by Bonferroni post hoc test. Kruskal-Wallis test for nonparametric data in $\boldsymbol{E}$. Scale bars: $\boldsymbol{B}, \boldsymbol{C}$, $50 \mu \mathrm{m} ; \boldsymbol{I}, 100 \mu \mathrm{m} ; \boldsymbol{E}, 200 \mu \mathrm{m} ; \boldsymbol{G}, 2 \mu \mathrm{m} ; \boldsymbol{H}, 1 \mu \mathrm{m}$. 

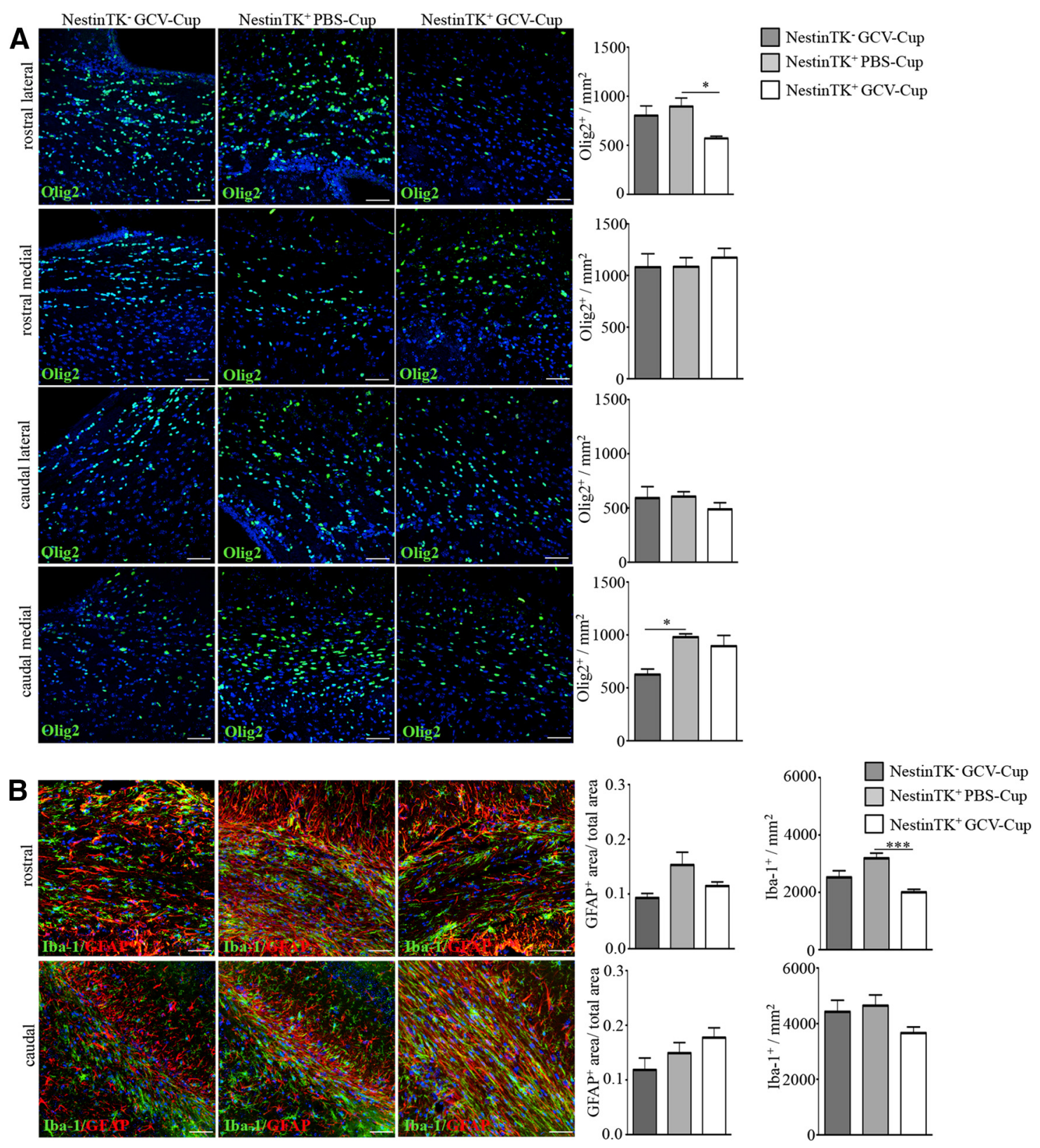

Figure 3. Effect of early eNPCs ablation on astrocytes, microglia and oligodendroglia. $A$, Representative images and quantification of oligodendrocyte precursors (Olig2 ${ }^{+}$, in green) in the rostral and caudal regions of $\mathrm{CC}$, at lateral and medial levels in NPC-ablated mice and controls at 6 weeks after the cuprizone diet. Graphs showing the number of 0 lig2 ${ }^{+}$cells. $n=6$ mice/group. $\boldsymbol{B}$, Representative images and quantification of astrocytes (GFAP, in red) and of microglia (Iba-1, in green) at the level of the rostral and caudal CC at 6 weeks after cuprizone treatment in NPC-ablated mice and controls. Graphs show the number of GFAP ${ }^{+}$and lba- $1^{+}$cells per area. $n=6$ mice/group. Nuclei counterstained by DAPI are in blue. Each bar represents the mean \pm SEM. ${ }^{*} p \leq 0.05$; ${ }^{* * *} p \leq 0.001$; one-way ANOVA followed by Bonferroni post hoc test. Scale bar, $50 \mu \mathrm{m}$.

BrdU, were significantly reduced as previously described for this transgenic mouse (Butti et al., 2012; Fig. 2B,C).

To follow longitudinally, in vivo, the damage of the CC, we performed MRI at the fourth and sixth week of cuprizone diet. On T2-weighted images, an increased signal of CC was observed (hyperintensity of the CC) at 4 weeks of cuprizone diet in all groups of mice when compared with mice being fed a standard diet (Fig. 2D). Mice with ablated eNPCs did not show further alterations of the T2 signal compared with cuprizone-treated controls (Fig. 2D). We further considered the diffusivity measures, and, as expected in all cuprizone-fed mice, we observed a significant decrease of fractional anisotropy, mean diffusivity, and axial diffusivity compared with mice fed a standard diet at 4 weeks. At the sixth week of cuprizone treatment, values of frac- tional anisotropy and axial diffusivity were still altered, while the mean diffusivity almost returned to average values, suggesting that demyelination was still ongoing. However, we did not identify significant changes between cuprizone-treated mice, ablated or not (Fig. 2D).

To further analyze whether eNPC ablation could influence the CC damage at the end of the cuprizone diet, demyelination was assayed by Luxol fast blue staining on coronal sections from mice killed at the sixth week (Fig. 2E). Severe myelin loss was observed in mice receiving cuprizone, both in the caudal and the rostral CC. However, eNPC-ablated mice (NestinTK ${ }^{+}$GCV-Cup) did not show increased demyelination when compared with their controls (NestinTK ${ }^{-}$GCV-Cup and NestinTK ${ }^{+}$PBS-Cup; Fig. $2 E)$. Furthermore, we evaluated axonal damage using Biel- 
A

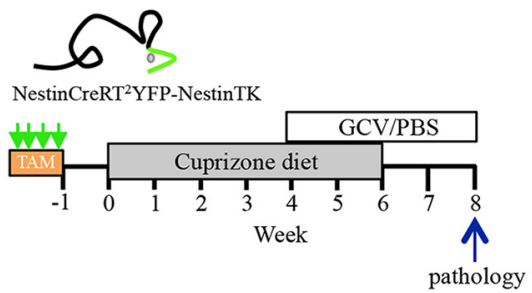

D
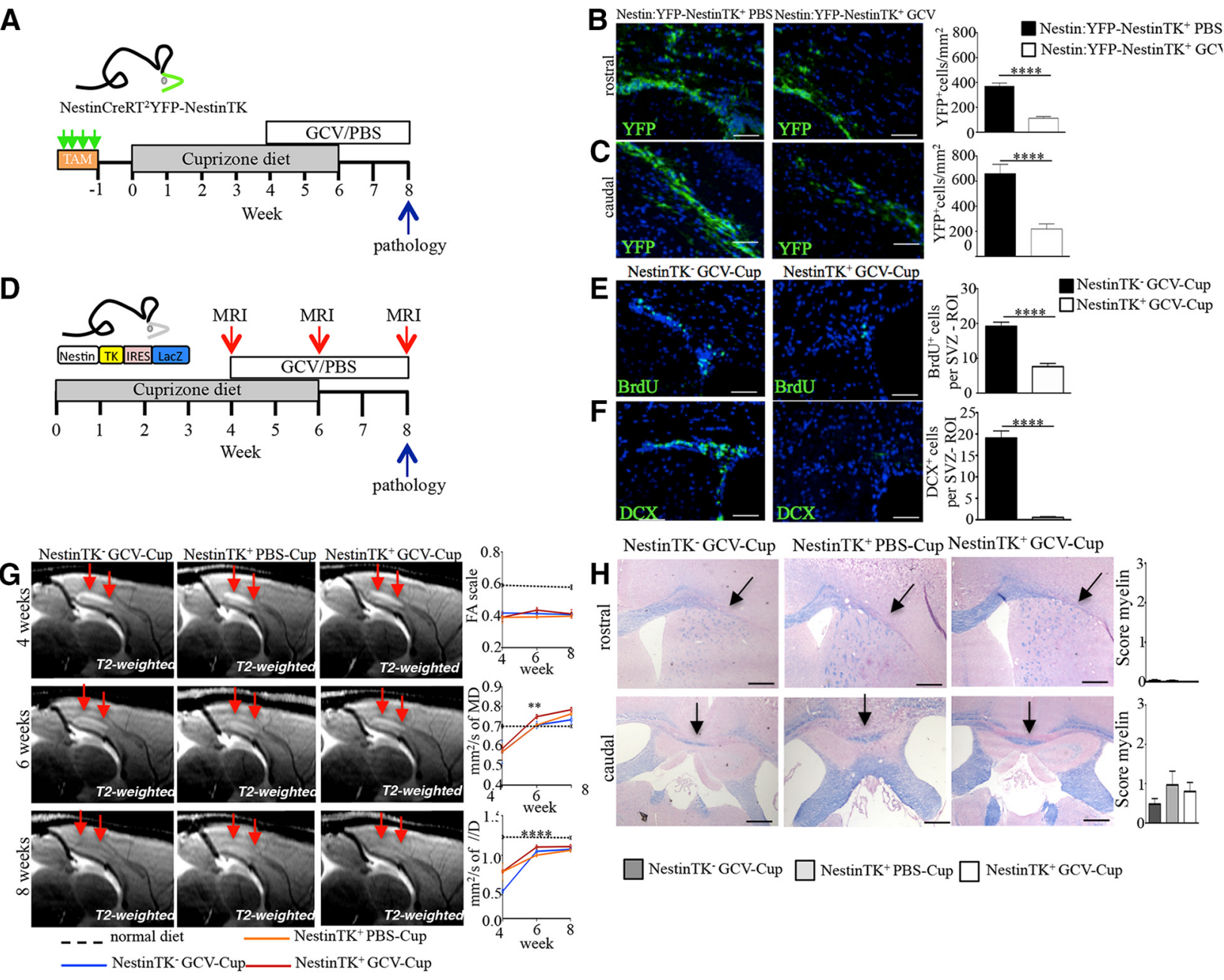

NestinTK- GCV-Cup NestinTK ${ }^{+}$PBS-Cup NestinTK ${ }^{+}$GCV-Cup
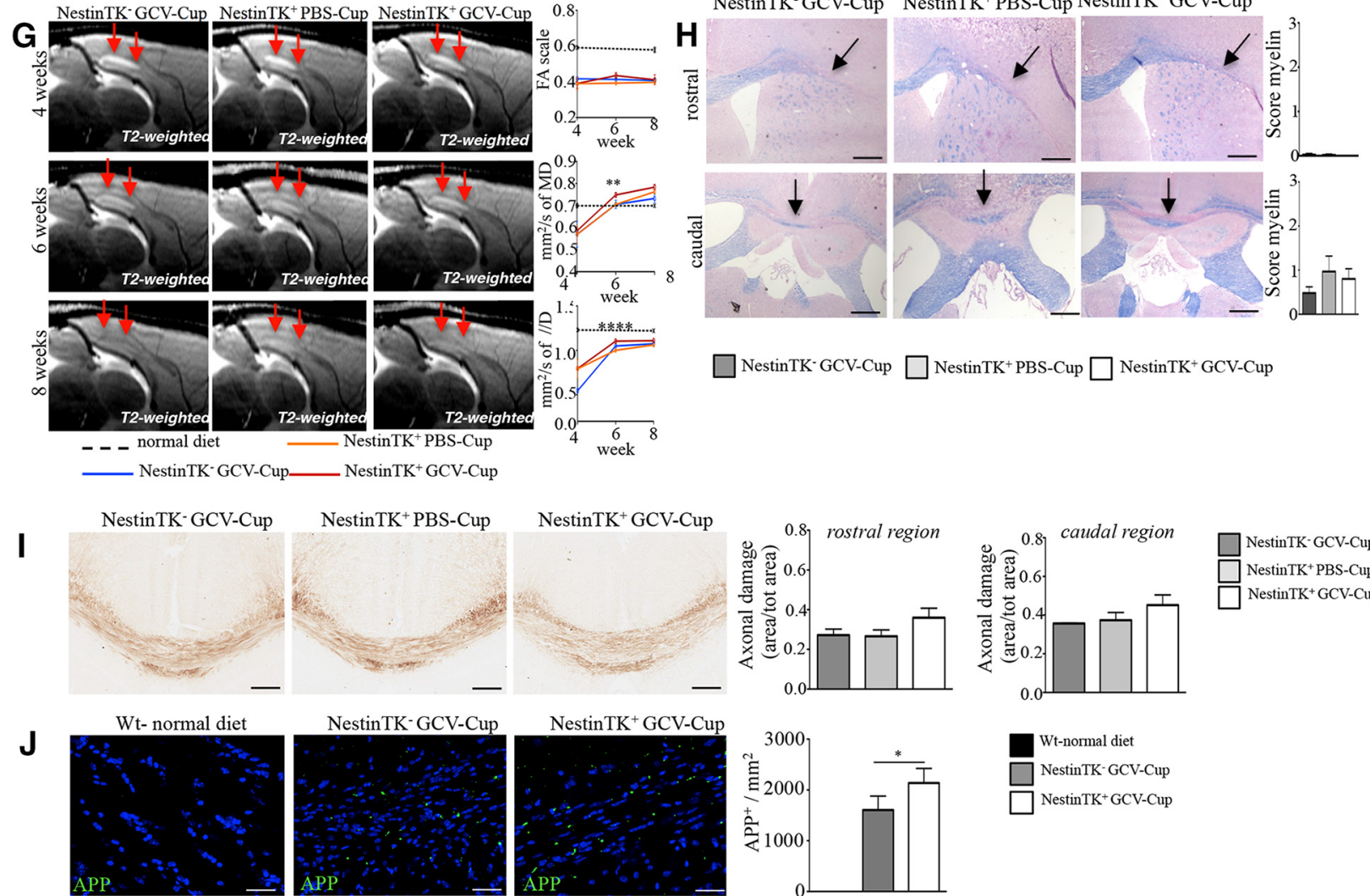

NestinTK ${ }^{+}$PBS-Cup
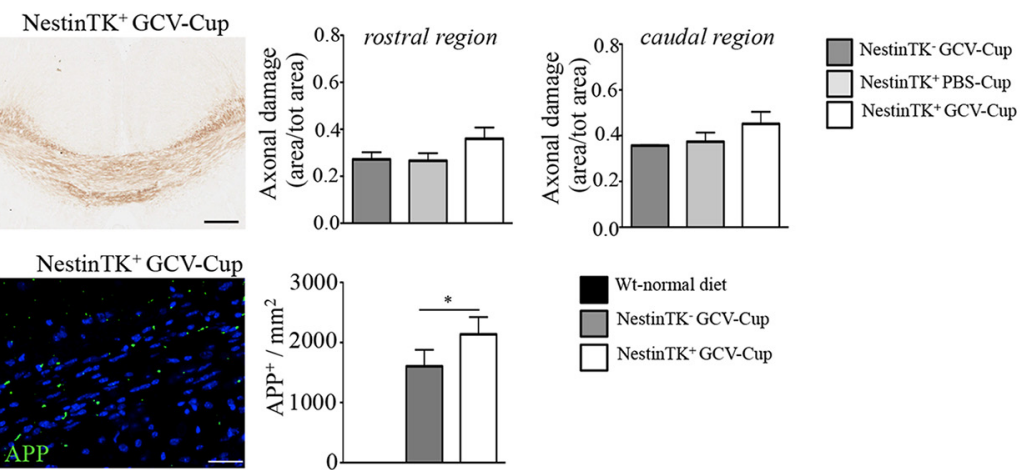

\section{K}

Wt-normal diet
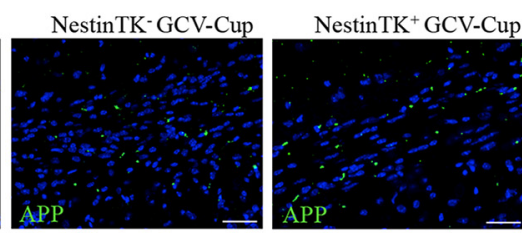

NestinTK-GCV-Cup

NestinTK ${ }^{+}$GCV-Cup
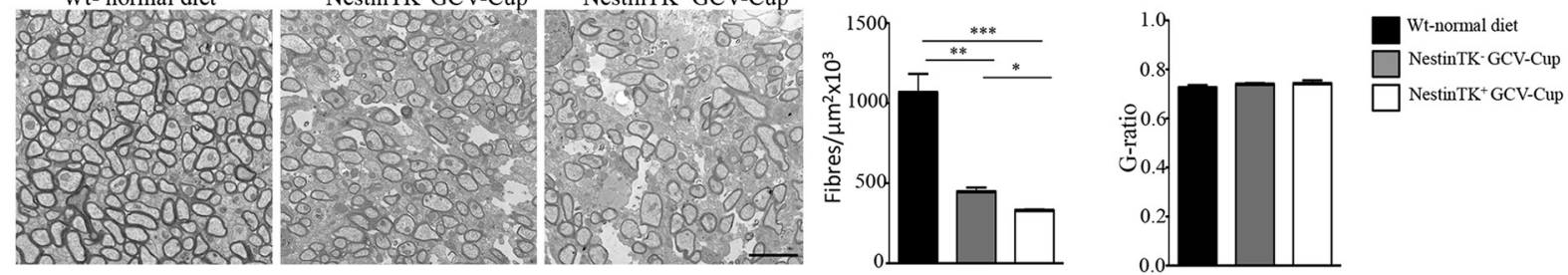

Figure 4. Delayed ablation of NPCs in cuprizone diet-treated mice to study remyelination. $A$, Experimental design to monitor the effective ablation of eNPCs using the NestinCreRT ${ }^{2} Y F P$ reporter mouse crossed with the NestinTK mouse, thus obtaining the Nestin:YFP-NestinTK mice. Mice were treated with tamoxifen by gavage for $4 \mathrm{~d}$, and then the demyelination of the CC was induced by feeding for 6 weeks of the cuprizone diet. Mice were treated with GCV or PBS from the fourth week to the eighth week to ablate SVZ-eNPCs during the remyelination phase (late phase) and histology performed at the eighth week. $\boldsymbol{B}, \boldsymbol{C}$, Quantification of YFP ${ }^{+}$cells (in green) in the rostral $(\boldsymbol{B})$ and caudal ( $\boldsymbol{C}$ ) regions of the CC. $n=4$ animals/group. $\boldsymbol{D}$, Experimental design to ablate eNPCs using the NestinTK mouse in the remyelination phase. Mice were treated either with GCV or PBS from the fourth week to the eighth week to ablate SVZ-eNPCs during the remyelination phase (late phase). MRI was performed at 4, 6, and 8 weeks to monitor the (C damage, and histology was performed at the eighth week. $\boldsymbol{E}, \boldsymbol{F}$, Quantification of transit amplifying cells, labeled by BrdU (in green; $\boldsymbol{E}$ ) and neuroblasts (DCX in green; $\boldsymbol{F}$ ) in the SVZ of NestinTK ${ }^{+} \mathrm{GCV}$ mice and in control mice. $n=3-6$ mice/group. $\mathbf{G}$, Analysis of $\mathrm{CC}$ damage by MRI. Representative sagittal T2-weighted images acquired in vivo at the fourth, sixth, and eighth week. Arrows indicate the lesion of the corpus callosum and its evolution over time. Analysis of DTI parameters: (Figure legend continues.) 
schowsky staining and observed no significant difference among the experimental groups (Fig. $2 F$ ). To morphologically validate myelin loss, we performed ultrastructural analysis of the caudal region of CC at the sixth week by EM. While cuprizone induced, as expected (Schultz et al., 2017), axonal loss and demyelination (Fig. $2 G$ ), we did not find a difference regarding the density of myelinated and unmyelinated axons between cuprizone-treated NPC-ablated and nonablated mice (Fig. $2 G$ ).

We next investigated the number of oligodendrocyte precursors in the lesioned corpus callosum in the absence of eNPCs (Fig. $3 A$ ). Thus, we analyzed the number of OPCs at the sixth week in the rostral region near the SVZ and the caudal region (Fig. 3A). Interestingly, we found that eNPC-ablated mice had a decreased number of Olig2 ${ }^{+}$cells in the rostral lateral region, compared with cuprizone-treated control mice (Fig. $3 A$ ). We further assessed the influence of eNPC depletion on astrogliosis and microgliosis concurrently occurring with demyelination in the cuprizone model (Gudi et al., 2014). Therefore, we determined the density of astrocytes by labeling brain sections either for GFAP or ALDH1L1 (data not shown). With both types of staining, no significant difference in terms of astrocytic reaction in the three treatment groups was observed (Fig. 3B).

Interestingly, microgliosis, evaluated by Ionized calcium binding adaptor molecule 1 (Iba-1) staining, was found to be slightly, but significantly, reduced in the rostral area of eNPCablated mice (Fig. 3B). We also performed staining to characterize microglia activation, using activation markers such as CD68 and CD11c. CD68 was significantly increased in eNPC-ablated mice $\left(199.7\right.$ vs $132.6 \mathrm{CD}^{+} 8^{+}$cells $/ \mathrm{mm}^{2}, \quad p=0.01$; in NestinTK ${ }^{+}$GCV-Cup and NestinTK ${ }^{-}$GCV-Cup mice, respectively), despite the overall reduction of Iba- $1^{+}$cells observed. Moreover, also the phagocytic marker $\mathrm{CD} 11 \mathrm{c}$ was found to be increased in mice devoid of eNPC $\left(26.1 \%\right.$ vs $19.3 \%$ of CD $11 \mathrm{c}^{+} \mathrm{Iba}-$ $1^{+}$cells/ Iba- $1^{+}$cells; $p=0.027$; in NestinTK ${ }^{+}$GCV-Cup and NestinTK ${ }^{-}$GCV-Cup mice, respectively). Finally, we found no significant difference evaluating $\mathrm{LPL}^{+}$microglia cells $(23.4 \%$ vs $29.8 \%$ of $\mathrm{LPL}^{+} \mathrm{Iba}-1^{+}$cells/Iba- $1^{+}$cells; $p=0.25 ;$ in NestinTK ${ }^{+}$GCV-Cup and NestinTK ${ }^{-}$GCV-Cup mice, respectively), a marker that has been recently described to indicate a subset of "reparative microglia" (Bruce et al., 2018). To highlight a possible contribution from infiltrating monocytes, we also stained for Ly6C but could only observe at 6 weeks very few infiltrating Ly6C ${ }^{+}$cells (data not shown), which in cuprizone-

$\leftarrow$

(Figure legend continued.) fractional anisotropy, mean diffusivity, and axial diffusivity. $n=3$ animals/group. $\boldsymbol{H}$, Representative brain sections of Luxol fast blue (LFB) staining are shown in the rostral and caudal regions of $\mathrm{CC}$ in NestinTK ${ }^{-}$and NestinTK ${ }^{+}$mice treated with either PBS or GCV 2 weeks after the end of the cuprizone diet. The entity of demyelination was judged by scoring LFB. Arrows indicate the lesion of the corpus callosum. $n=8 \mathrm{mice} / \mathrm{group} .0=$ complete myelination, 3 = complete demyelination. $I$, Representative brain sections of Bielschowsky staining are shown in the caudal region of $\mathrm{CC}^{\text {in NestinTK }}{ }^{-}$and NestinTK ${ }^{+}$mice treated with either PBS or GCV at 8 weeks. The entity of axonal damage was evaluated in the rostral and caudal regions by Bielschowsky staining. $n=3$ mice/group. $J$, Representative brain sections of APP staining are shown in the caudal region of $\mathrm{CC}$ in mice treated with normal diet, in NestinTK ${ }^{-}$and NestinTK ${ }^{+}$mice treated with GCV for 2 weeks at 8 weeks. Graph shows the number of $\mathrm{APP}^{+}$cells at 8 weeks in the caudal region after the cuprizone diet. $n=3$ mice/ group. $\boldsymbol{K}$, Representative electron micrographs of the caudal $\mathrm{CC}$ of mice treated with normal diet, of NestinTK ${ }^{-}$GCV-Cup and NestinTK ${ }^{+}$GCV-Cup mice at the eighth week. Graphs show the axonal density and the g-ratio of myelinated axons in mice fed with a normal diet and in NestinTK ${ }^{-}$GCV-Cup and NestinTK ${ }^{+}$GCV-Cup mice. $n=3$ mice/group. Each bar represents the mean \pm SEM. ${ }^{*} p \leq 0.05 ;{ }^{* *} p \leq 0.01 ;{ }^{* * *} p \leq 0.001 ;{ }^{* * * *} p \leq 0.0001$ (unpaired $t$ test in $B, C$, $\boldsymbol{E}$, and $\boldsymbol{F}$; one-way ANOVA followed by Bonferroni post hoc test in $\boldsymbol{G}, \boldsymbol{J}$, and $\boldsymbol{K}$ ). Kruskal-Wallis test for nonparametric data in $\boldsymbol{H}$. Scale bars: $\boldsymbol{B}, \boldsymbol{C}, \boldsymbol{E}, \boldsymbol{F}, 50 \mu \mathrm{m} ; \boldsymbol{I}, 100 \mu \mathrm{m} ; \boldsymbol{H}, 200 \mu \mathrm{m} ; \boldsymbol{K}, 2 \mu \mathrm{m}$. induced lesions represent a minority of inflammatory brain cells (Lampron et al., 2015).

\section{SVZ-eNPC ablation does not alter late-occurring remyelination and is accompanied by increased axonal loss}

Since after early eNPC ablation we did not observe a variation in the damaged entity and the demyelination of the CC after cuprizone injury, we decided to investigate the role of eNPC in the late phase of remyelination, namely at 8 weeks.

Mice were maintained on the cuprizone diet for 6 weeks and allowed to recover for 2 weeks after the end of cuprizone diet. eNPC ablation was induced by administering GCV at 4 weeks after cuprizone starting and up to the eighth week (Fig. $4 D$, experimental scheme).

First of all, we verified the efficacy of SVZ-eNPC ablation induced by GCV administration using triple transgenic mice Nestin-CreER ${ }^{\mathrm{T} 2}$-YFP-NestinTK (called Nestin:YFP-NestinTK; Fig. 4A), in which SVZ-derived eNPCs and its progeny can be traced. Using this mouse line, we found that at 8 weeks $\sim 70 \%$ of $\mathrm{YFP}^{+}$cells, migrated either to the rostral or caudal region of the CC, were reduced upon GCV treatment (Fig. 4B,C). At 8 weeks, neuroblast (DCX) and BrdU positive transient amplifying cells are still absent and the SVZ is not repopulated (Fig. 4E, F).

Even in this experimental paradigm, we monitored the CC lesions by MRI scans at the fourth, sixth, and eighth weeks. We observed in all treatment groups that the lesion in the CC (at 4 weeks) steadily recovers over time; mean and axial diffusivity returned to normal values at 8 weeks while fractional anisotropy was still reduced. This indicates that the remyelination had occurred, but that the fiber density remains low if compared with healthy mice. The ablation of eNPCs even in the late phase of the disease apparently did not influence the CC damage, as evaluated by MRI (Fig. $4 G$ ).

Through Luxol fast blue staining, we observed residual myelin damage in the rostral and caudal region of CC, but no difference between treatment groups (Fig. 4H). Examining the axonal damage by Bielschowsky staining did not reveal significant differences between groups, despite a small trend of increased damage in eNPC-ablated mice (Fig. 4I). We also evaluated by immunofluorescence the presence of amyloid precursor protein (APP) as marker of axonal damage (Schultz et al., 2017). Cuprizonetreated eNPC-ablated mice showed an increase of $\mathrm{APP}^{+}$compared with nonablated mice, while non-cuprizone-treated mice showed no axonal damage (Fig. $4 J$ ).

To evaluate thoroughly axonal damage and remyelination, we next performed ultrastructural analysis by EM. This analysis unveiled a significant fiber density reduction upon cuprizone treatment in eNPC-ablated mice compared with nonablated cuprizonetreated mice. Contrariwise, the efficacy of remyelination, quantified by measuring the G-ratio on EM images, was found comparable between eNPC-ablated mice and controls. (Fig. 4L).

We finally assessed quantitatively the number of oligodendrocytes, astrocytes, and microglia, in the rostral and caudal region of the CC (separating lateral and medial areas; Fig. $5 A, B$ ). We found that eNPC-ablated mice did not show any difference, compared with controls, in the number of Olig2 ${ }^{+}$cells (Fig. 5A). Considering mature oligodendrocytes instead, we observed a reduction of $\mathrm{APC}^{+}$cells in the medial caudal region of NestinTK ${ }^{+}$ mice (Fig. 5B). Finally, we verified astrogliosis and microgliosis but found at this stage of the disease no significant changes between the treatment groups (Fig. $5 C$ ). 
A

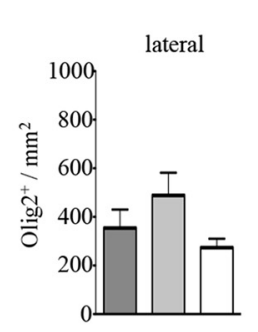

rostral region

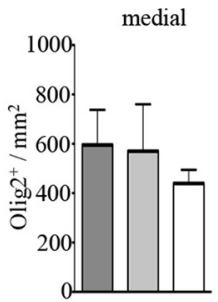

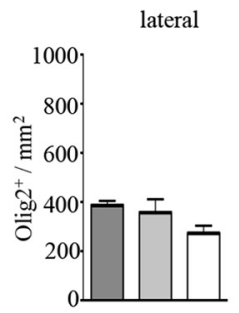

caudal region

\section{B}
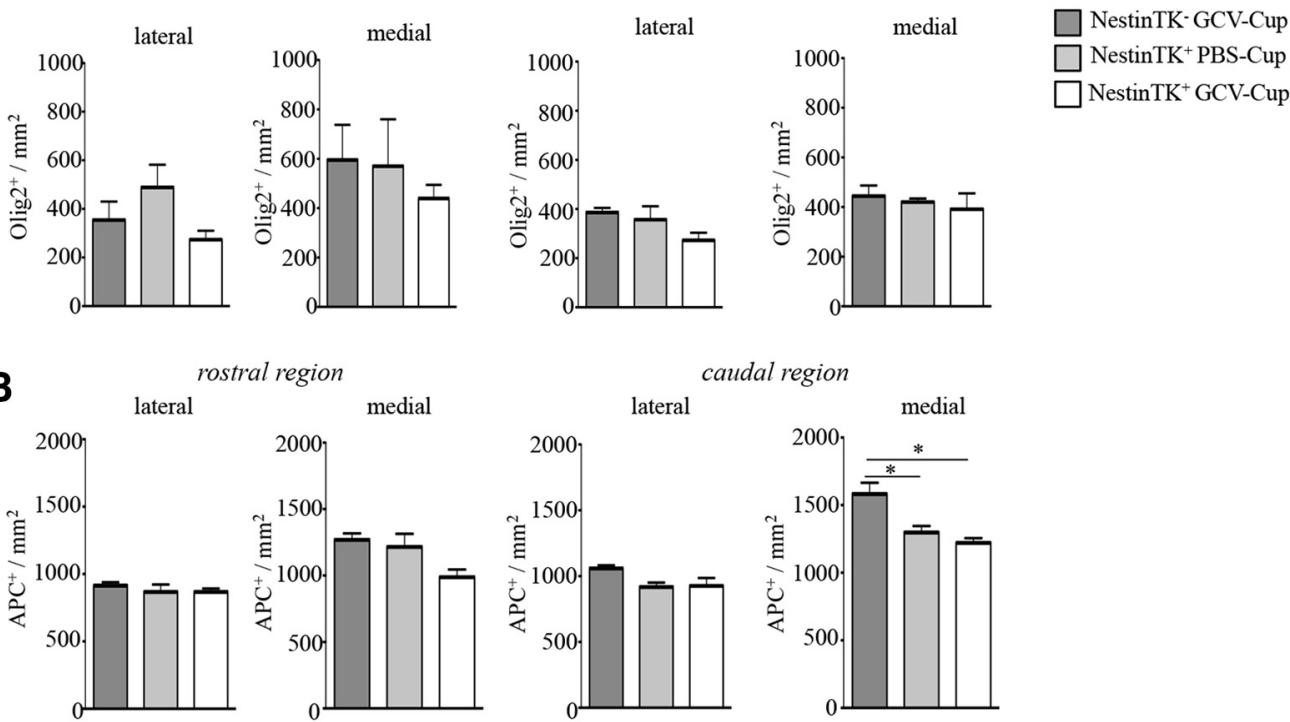

caudal region
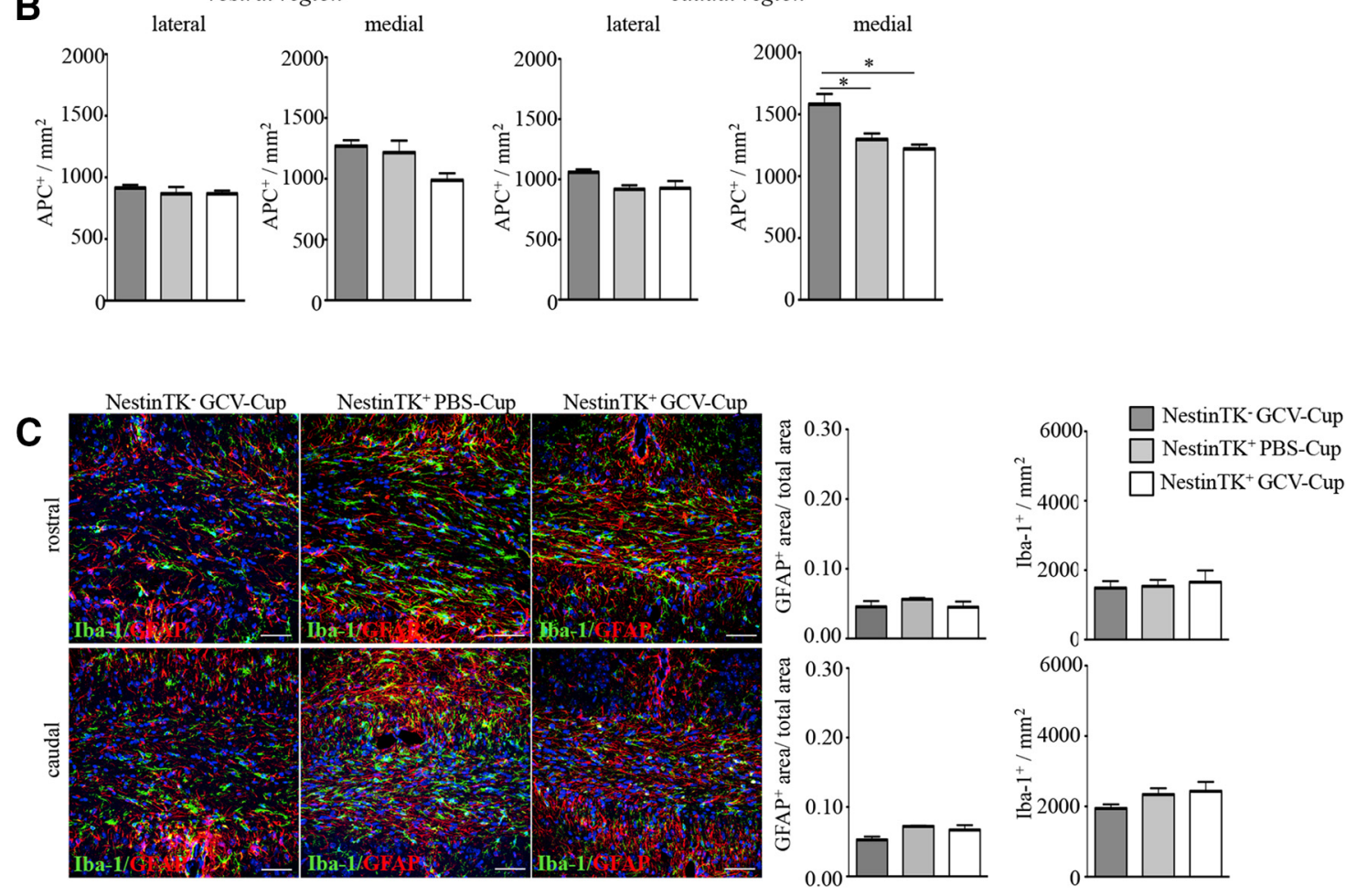

Figure 5. Effect of late eNPCs ablation on oligodendrocytes, astrogliosis, and microgliosis. $A$, Quantification of Olig2 ${ }^{+}$cells at 8 weeks in rostral and caudal region after the cuprizone diet. $n=$ 6 mice/group. $B$, Graphs show the number of $A C^{+}$cells at 8 weeks in rostral and caudal regions after the cuprizone diet. $n=6$ mice/group. $C$, Representative images and quantification of astrocytes (GFAP, in red) and of microglia (Iba-1, in green) at the level of the rostral and caudal CC at 8 weeks in NPC-ablated mice and controls. Graphs show the number of GFAP ${ }^{+}$and Iba- ${ }^{+}$cells per area. $n=6$ mice/group. Each bar represents the mean \pm SEM. ${ }^{*} p \leq 0.05$; one-way ANOVA followed by Bonferroni post hoc test. Scale bar, $50 \mu \mathrm{m}$.

\section{Discussion}

Multiple sclerosis is a chronic demyelinating disease of the CNS. Chronic demyelination leads to axonal loss that causes a progressive clinical decline. In some patients with MS, remyelination occurs early in disease development. However, remyelination fails in many cases and the majority of chronic MS plaques remain demyelinated (Franklin and Ffrench-Constant, 2008). Remyelination is essential for the return of neuronal function and to prevent axonal loss (Hartley et al., 2014). The poor remyelination in MS and the derived loss of axonal integrity pose remyelination as a critical therapeutic objective. Identifying potential targets requires a thorough understanding of the cellular and molecular mechanisms of remyelination. Recent studies have demonstrated that besides the endogenous population of pOPCs, eNPCs within the SVZ represent an alternative source of progenitors for remyelination (Menn et al., 2006; Nait-Oumesmar et al., 2007; Ortega et al., 2013) that is mobilized after a demyelinated damage (Jablonska et al., 2010; Xing et al., 2014; Brousse et al., 2015). ENPC-induced remyelination can also be pharmacologically increased as recently reported (Samanta et al., 2015).
Nonetheless, in experimental MS it is difficult to dissect the precise role of eNPCs in the remyelination process, because the autoimmune $\mathrm{T}$ cell-mediated reaction coexists within demyelinating areas and affecting NPC functions. It has been shown that neuroinflammation induces a profound dysfunction of the SVZ niche, which, in turn, restrains eNPCs proliferation capacity (Pluchino et al., 2008; Rasmussen et al., 2011). Therefore, to understand the precise role of eNPCs during the remyelination process, we focused our attention on the role of eNPCs in the cuprizone-induced toxic demyelination model that is also accompanied by axonal loss (Schultz et al., 2017). The cuprizone model is indeed a very reproducible and widely used model that has resulted over the years in being an invaluable tool for studying mechanisms of acquired demyelination and remyelination (Kipp et al., 2009; Gudi et al., 2014). Moreover, the model precisely distinguishes between demyelination and remyelination mechanisms and dissects the specific function of eNPCs without the confounding presence of overt T-cell infiltrates.

In the first set of experiments, we aimed at investigating the mobilization and the fate specification of SVZ-derived NPCs. In agreement with Xing et al. (2014), we observed the recruit- 
ment of eNPC-derived cells in the CC with lesions induced by cuprizone with a different regionalization. We focused our attention on the percentage of Olig2-expressing YFP to understand how many Olig2 cells derived from the SVZ-eNPC, and in accordance with the study by Brousse et al. (2015), we showed that only a low number of oligodendrocytes are derived from eNPCs at the eighth week ( 2 weeks after the end of cuprizone diet). These data indicate that eNPCs derived from SVZ can generate new oligodendrocytes in this demyelinating model, but also support the concept that parenchymal OPCs are mainly involved in the remyelination process since they are homogeneously distributed in the entire CC from the rostral to the caudal region. Considering the SVZ position, we thought to find a significant difference in the rostral and not in the caudal region, since the first is close to the SVZ (Stidworthy et al., 2003; Wu et al., 2008; Xie et al., 2010; Steelman et al., 2012). We thus investigated in more detail the precise role of eNPCs in cuprizone-induced demyelination and remyelination process using a transgenic mouse model (NestinTK) that allows the selective ablation of SVZ-eNPCs upon ganciclovir treatment (Butti et al., 2012). First of all, we chose to ablate eNPCs from the first week to the fifth week during the cuprizone diet, since during these weeks they may exert an influence on the process of microgliosis, astrogliosis, and oligodendrogenesis. Microgliosis has been shown to initiate within the first 2 weeks of cuprizone treatment. During this time, astrocytes display morphological changes, and after 3 weeks their number significantly increases (Gudi et al., 2014). After 3-5 weeks, parallel to the myelin clearance in CC, local and migrated OPCs start to proliferate within the lesions (Gudi et al., 2014). We assessed the mice at the sixth week after starting of cuprizone diet, when the demyelination is complete (Gudi et al., 2014). We evaluated the myelin damage of $\mathrm{CC}$, axonal loss, the astrogliosis, the number of microglia, and the generation of new myelinating cells. Our results indicate that at this time point SVZ-derived NPCs ablation influences neither the cuprizone-mediated myelin damage, nor the number of demyelinated axons, nor the extent of astrogliosis. However, eNPC ablation was associated with increased activation of Iba- $1^{+}$cells, microglia. It is known that eNPCs can regulate microglia density and function as well as its activity by VEGF secretion (Mosher et al., 2012). Thus, we can speculate that in this lesion model the lack of eNPC-secreted growth factors, such as VEGF (Mosher et al., 2012; Bacigaluppi et al., 2016) or TGF- $\beta$ (De Feo et al., 2017), might be responsible for the increased microglia activation.

To investigate the effect of eNPCs on remyelination, we ablated eNPCs in the last phase of the cuprizone diet and up to 2 weeks after cuprizone withdrawal (from the fourth to the eighth week). CC remyelination, astrogliosis, and microgliosis were not diversely affected in eNPC-ablated mice. We cannot exclude, as suggested by Xing et al. (2014), that an inverse relationship between eNPC- and pOPC-derived oligodendrocytes might exist, meaning that the presence of one may have an inhibitory effect on the other, in terms of efficacy during the remyelination process.

Interestingly, in eNPC-ablated mice we found instead a decreased density of the axonal fibers located within the caudal CC, showing that the absence of eNPCs might partially exacerbate axonal loss after cuprizone-induced damage.

Altogether our study shows that SVZ-eNPCs mobilized after the first demyelinating period, partially contribute to replenish the pool of oligodendrocyte precursors and are able to reduce the activation state of microglia. This latter effect, in turn, might promote partial neuroprotection of corpus callosum axons at later stages. In other diseases such as stroke, epilepsy, and gliomas, we and others have demonstrated that eNPCs can sense inflammatory signals and reduce the tissue damage by releasing neuroprotective molecules (Martino et al., 2014), thus contributing to maintenance of CNS homeostasis. We thus propose that such an indirect repairing mechanism, instructed by eNPCs, might also operate in the cuprizone-induced demyelination model.

\section{References}

Acs P, Kipp M, Norkute A, Johann S, Clarner T, Braun A, Berente Z, Komoly S, Beyer C (2009) 17 $\beta$-estradiol and progesterone prevent cuprizone provoked demyelination of corpus callosum in male mice. Glia 57: 807-814.

Aguirre A, Dupree JL, Mangin JM, Gallo V (2007) A functional role for EGFR signaling in myelination and remyelination. Nat Neurosci 10: 990-1002.

Bacigaluppi M, Russo GL, Peruzzotti-Jametti L, Rossi S, Sandrone S, Butti E, De Ceglia R, Bergamaschi A, Motta C, Gallizioli M, Studer V, Colombo E, Farina C, Comi G, Politi LS, Muzio L, Villani C, Invernizzi RW, Hermann DM, Centonze D, et al. (2016) Neural stem cell transplantation induces stroke recovery by upregulating glutamate transporter GLT-1 in astrocytes. J Neurosci 36:10529-10544.

Barkhof F, Bruck W, De Groot CJ, Bergers E, Hulshof S, Geurts J, Polman CH, van der Valk P (2003) Remyelinated lesions in multiple sclerosis: magnetic resonance image appearance. Arch Neurol 60:1073-1081.

Brousse B, Magalon K, Durbec P, Cayre M (2015) Region and dynamic specificities of adult neural stem cells and oligodendrocyte precursors in myelin regeneration in the mouse brain. Biol Open 4:980-992.

Bruce KD, Gorkhali S, Given K, Coates AM, Boyle KE, Macklin WB, Eckel RH (2018) Lipoprotein lipase is a feature of alternatively activated microglia and may facilitate lipid uptake in the CNS during demyelination. Front Mol Neurosci 11:57.

Butti E, Bacigaluppi M, Rossi S, Cambiaghi M, Bari M, Cebrian Silla A, Brambilla E, Musella A, De Ceglia R, Teneud L, De Chiara V, D’Adamo P, Garcia-Verdugo JM, Comi G, Muzio L, Quattrini A, Leocani L, Maccarrone M, Centonze D, Martino G (2012) Subventricular zone neural progenitors protect striatal neurons from glutamatergic excitotoxicity. Brain 135:3320-3335.

De Feo D, Merlini A, Brambilla E, Ottoboni L, Laterza C, Menon R, Srinivasan S, Farina C, Garcia Manteiga JM, Butti E, Bacigaluppi M, Comi G, Greter M, Martino G (2017) Neural precursor cell-secreted TGF-beta2 redirects inflammatory monocyte-derived cells in CNS autoimmunity. J Clin Invest 127:3937-3953.

Franklin RJ, Ffrench-Constant C (2008) Remyelination in the CNS: from biology to therapy. Nat Rev Neurosci 9:839-855.

Garcia AD, Doan NB, Imura T, Bush TG, Sofroniew MV (2004) GFAPexpressing progenitors are the principal source of constitutive neurogenesis in adult mouse forebrain. Nat Neurosci 7:1233-1241.

Goldman S (2005) Stem and progenitor cell-based therapy of the human central nervous system. Nat Biotechnol 23:862-871.

Gonzalez-Perez O, Alvarez-Buylla A (2011) Oligodendrogenesis in the subventricular zone and the role of epidermal growth factor. Brain Res Rev 67:147-156.

Gudi V, Gingele S, Skripuletz T, Stangel M (2014) Glial response during cuprizone-induced de- and remyelination in the CNS: lessons learned. Front Cell Neurosci 8:73.

Hartley MD, Altowaijri G, Bourdette D (2014) Remyelination and multiple sclerosis: therapeutic approaches and challenges. Curr Neurol Neurosci Rep 14:485.

Imayoshi I, Ohtsuka T, Metzger D, Chambon P, Kageyama R (2006) Temporal regulation of cre recombinase activity in neural stem cells. Genesis 44:233-238.

Jablonska B, Aguirre A, Raymond M, Szabo G, Kitabatake Y, Sailor KA, Ming GL, Song H, Gallo V (2010) Chordin-induced lineage plasticity of adult SVZ neuroblasts after demyelination. Nat Neurosci 13:541-550.

Kipp M, Clarner T, Dang J, Copray S, Beyer C (2009) The cuprizone animal model: new insights into an old story. Acta Neuropathol 118:723-736.

Lampron A, Larochelle A, Laflamme N, Préfontaine P, Plante MM, Sánchez MG, Yong VW, Stys PK, Tremblay MÈ, Rivest S (2015) Inefficient clear- 
ance of myelin debris by microglia impairs remyelinating processes. J Exp Med 212:481-495.

Martino G, Butti E, Bacigaluppi M (2014) Neurogenesis or non-neurogenesis: that is the question. J Clin Invest 124:970-973.

Menn B, Garcia-Verdugo JM, Yaschine C, Gonzalez-Perez O, Rowitch D, Alvarez-Buylla A (2006) Origin of oligodendrocytes in the subventricular zone of the adult brain. J Neurosci 26:7907-7918.

Mosher KI, Andres RH, Fukuhara T, Bieri G, Hasegawa-Moriyama M, He Y, Guzman R, Wyss-Coray T (2012) Neural progenitor cells regulate microglia functions and activity. Nat Neurosci 15:1485-1487.

Nait-Oumesmar B, Decker L, Lachapelle F, Avellana-Adalid V, Bachelin C, Baron-Van Evercooren A (1999) Progenitor cells of the adult mouse subventricular zone proliferate, migrate and differentiate into oligodendrocytes after demyelination. Eur J Neurosci 11:4357-4366.

Nait-Oumesmar B, Picard-Riera N, Kerninon C, Decker L, Seilhean D, Höglinger GU, Hirsch EC, Reynolds R, Baron-Van Evercooren A (2007) Activation of the subventricular zone in multiple sclerosis: evidence for early glial progenitors. Proc Natl Acad Sci U S A 104:4694-4699.

Noseworthy JH, Lucchinetti C, Rodriguez M, Weinshenker BG (2000) Multiple sclerosis. N Engl J Med 343:938-952.

Ortega F, Gascón S, Masserdotti G, Deshpande A, Simon C, Fischer J, Dimou L, Chichung Lie D, Schroeder T, Berninger B (2013) Oligodendrogliogenic and neurogenic adult subependymal zone neural stem cells constitute distinct lineages and exhibit differential responsiveness to wnt signalling. Nat Cell Biol 15:602-613.

Picard-Riera N, Decker L, Delarasse C, Goude K, Nait-Oumesmar B, Liblau R, Pham-Dinh D, Baron-Van Evercooren A (2002) Experimental autoimmune encephalomyelitis mobilizes neural progenitors from the subventricular zone to undergo oligodendrogenesis in adult mice. Proc Natl Acad Sci U S A 99:13211-13216.

Pluchino S, Quattrini A, Brambilla E, Gritti A, Salani G, Dina G, Galli R, Del Carro U, Amadio S, Bergami A, Furlan R, Comi G, Vescovi AL, Martino G (2003) Injection of adult neurospheres induces recovery in a chronic model of multiple sclerosis. Nature 422:688-694.

Pluchino S, Muzio L, Imitola J, Deleidi M, Alfaro-Cervello C, Salani G, Porcheri C, Brambilla E, Cavasinni F, Bergamaschi A, Garcia-Verdugo JM, Comi G, Khoury SJ, Martino G (2008) Persistent inflammation alters the function of the endogenous brain stem cell compartment. Brain 131:2564-2578.

Rasmussen S, Imitola J, Ayuso-Sacido A, Wang Y, Starossom SC, Kivisäkk P, Zhu B, Meyer M, Bronson RT, Garcia-Verdugo JM, Khoury SJ (2011) Reversible neural stem cell niche dysfunction in a model of multiple sclerosis. Ann Neurol 69:878-891.

Samanta J, Grund EM, Silva HM, Lafaille JJ, Fishell G, Salzer JL (2015) Inhibition of Gli1 mobilizes endogenous neural stem cells for remyelination. Nature 526:448-452.
Schultz V, van der Meer F, Wrzos C, Scheidt U, Bahn E, Stadelmann C, Brück W, Junker A (2017) Acutely damaged axons are remyelinated in multiple sclerosis and experimental models of demyelination. Glia 65: $1350-1360$.

Skripuletz T, Hackstette D, Bauer K, Gudi V, Pul R, Voss E, Berger K, Kipp M, Baumgärtner W, Stangel M (2013) Astrocytes regulate myelin clearance through recruitment of microglia during cuprizone-induced demyelination. Brain 136:147-167.

Srinivas S, Watanabe T, Lin CS, William CM, Tanabe Y, Jessell TM, Costantini F (2001) Cre reporter strains produced by targeted insertion of EYFP and ECFP into the ROSA26 locus. BMC Dev Biol 1:4.

Steelman AJ, Thompson JP, Li J (2012) Demyelination and remyelination in anatomically distinct regions of the corpus callosum following cuprizone intoxication. Neurosci Res 72:32-42.

Stidworthy MF, Genoud S, Suter U, Mantei N, Franklin RJ (2003) Quantifying the early stages of remyelination following cuprizone-induced demyelination. Brain Pathol 13:329-339.

Tagge I, O'Connor A, Chaudhary P, Pollaro J, Berlow Y, Chalupsky M, Bourdette D, Woltjer R, Johnson M, Rooney W (2016) Spatio-temporal patterns of demyelination and remyelination in the cuprizone mouse model. PLoS One 11:e0152480.

Tripathi RB, Rivers LE, Young KM, Jamen F, Richardson WD (2010) NG2 glia generate new oligodendrocytes but few astrocytes in a murine experimental autoimmune encephalomyelitis model of demyelinating disease. J Neurosci 30:16383-16390.

Wu QZ, Yang Q, Cate HS, Kemper D, Binder M, Wang HX, Fang K, Quick MJ, Marriott M, Kilpatrick TJ, Egan GF (2008) MRI identification of the rostral-caudal pattern of pathology within the corpus callosum in the cuprizone mouse model. J Magn Reson Imaging 27:446-453.

Xie M, Tobin JE, Budde MD, Chen CI, Trinkaus K, Cross AH, McDaniel DP, Song SK, Armstrong RC (2010) Rostrocaudal analysis of corpus callosum demyelination and axon damage across disease stages refines diffusion tensor imaging correlations with pathological features. J Neuropathol Exp Neurol 69:704-716.

Xing YL, Röth PT, Stratton JA, Chuang BH, Danne J, Ellis SL, Ng SW, Kilpatrick TJ, Merson TD (2014) Adult neural precursor cells from the subventricular zone contribute significantly to oligodendrocyte regeneration and remyelination. J Neurosci 34:14128-14146.

Zawadzka M, Rivers LE, Fancy SP, Zhao C, Tripathi R, Jamen F, Young K, Goncharevich A, Pohl H, Rizzi M, Rowitch DH, Kessaris N, Suter U, Richardson WD, Franklin RJ (2010) CNS-resident glial progenitor/ stem cells produce schwann cells as well as oligodendrocytes during repair of CNS demyelination. Cell Stem Cell 6:578-590.

Zhang J, Jones MV, McMahon MT, Mori S, Calabresi PA (2012) In vivo and ex vivo diffusion tensor imaging of cuprizone-induced demyelination in the mouse corpus callosum. Magn Reson Med 67:750-759. 